\title{
Long-lasting memory of jasmonic acid-dependent immunity requires DNA demethylation and ARGONAUTE1
}

\section{Samuel Wilkinson}

University of Sheffield https://orcid.org/0000-0002-4908-8766

Amos Muench

University of Sheffield

Robert Wilson

University of Sheffield https://orcid.org/0000-0001-9010-1363

Kourosh Hooshmand

Aarhus University

Michael Henderson

University of Sheffield

\section{Emma Moffat}

University of Sheffield https://orcid.org/0000-0002-8559-2142

Joost Stassen

University of Sheffield https://orcid.org/0000-0001-5483-325X

Ana López Sánchez

University of Sheffield

Inge Fomsgaard

Aarhus University

Paal Krokene

Norwegian Institute for Bioeconomy Research

Melissa Magerøy

Norwegian Institute for Bioeconomy Research

Jurriaan Ton ( $\boldsymbol{\nabla}$ j.ton@sheffield.ac.uk)

University of Sheffield https://orcid.org/0000-0002-8512-2802

\section{Article}

Keywords: Arabidopsis thaliana, jasmonic acid, herbivory

Posted Date: August 6th, 2021

DOl: https://doi.org/10.21203/rs.3.rs-148432/v1 
License: (c) (i) This work is licensed under a Creative Commons Attribution 4.0 International License. Read Full License 
4 Wilkinson SW ${ }^{1}$, Muench $\mathrm{A}^{1}$, Wilson RS ${ }^{1}$, Hooshmand $\mathrm{K}^{2}$, Henderson MA ${ }^{1}$, Moffat EK ,

5 Stassen $\mathrm{JHM}^{1}$, López Sánchez $\mathrm{A}^{1}$, Fomsgaard $\mathrm{IS}^{2}$, Krokene $\mathrm{P}^{3}$, Mageroy $\mathrm{MH}^{3}$ and Ton $\mathrm{J}^{1, *}$ 6

$7 \quad{ }^{1}$ Plants, Photosynthesis and the Rhizosphere Cluster, School of Biosciences, Institute for 8 Sustainable Food, The University of Sheffield, Sheffield S10 2TN, United Kingdom

92 Department of Agroecology, Aarhus University, DK-4200 Slagelse, Denmark

$10{ }^{3}$ Division for Biotechnology and Plant Health, Norwegian Institute of Bioeconomy Research 11 (NIBIO), 1433 Ås, Norway

$12{ }^{*}$ Correspondence: j.ton@sheffield.ac.uk 


\section{ABSTRACT}

14 Stress can alter important plant life-history traits. Here, we report the long-term effects of the 15 stress hormone jasmonic acid (JA) on the defence phenotype, transcriptome and DNAmethylome of Arabidopsis. Three weeks after transient JA signalling activity, 5-week-old plants retained induced resistance (IR) against herbivory but showed enhanced susceptibility to necrotrophic and biotrophic pathogens. Transcriptome analysis of these plants revealed priming and/or up-regulation of JA-dependent defence genes but repression of ethyleneand salicylic acid-dependent genes. Long-term JA-IR against herbivory was associated with shifts in glucosinolate composition and required MYC2/3/4 transcription factors, DNA (de)methylation pathways and the small RNA (sRNA)-binding protein ARGONOUTE1 (AGO1). Although methylome analysis did not reveal consistent changes in DNA methylation near MYC2/3/4-controlled genes, JA-treated plants were specifically enriched with hypomethylated ATREP2 transposable elements (TEs), while ATREP2-derived sRNAs showed increased association with AGO1. Our results indicate that AGO1-associated sRNAs from hypomethylated ATREP2 TEs trans-regulate long-lasting memory of JAdependent immunity. 


\section{INTRODUCTION}

To resist pests and diseases, plants have evolved wide-ranging strategies which unfold over different timescales ${ }^{1}$. Pattern-triggered immunity $(\mathrm{PTI})$ is an immediate immune response that protects against most attackers. However, specialised pests and diseases can suppress PTI, enabling them to initiate a parasitic interaction with their hosts ${ }^{2-4}$. The residual basal resistance is too weak to arrest specialised attackers but contributes to slowing down their colonisation ${ }^{5}$. Moreover, specific environmental signals can augment basal resistance. This so-called induced resistance (IR) is mediated by prolonged upregulation and/or priming of PTI-related defences ${ }^{1}$, as PTI, basal resistance and IR share similar signalling pathways ${ }^{3,5,6}$. The defence hormones salicylic acid (SA) and jasmonic acid (JA) play central roles in these pathways $^{6,7}$. While SA-dependent defences are mostly effective against (hemi-)biotrophic pathogens, JA activates defences against both necrotrophic pathogens and herbivores ${ }^{3,7,8}$.

The immediate effects of JA signalling on defence gene expression are well documented. In Arabidopsis thaliana (Arabidopsis), bio-active JA-isoleucine (JA-lle) stimulates binding of the F-box protein COI1 to JAZ repressor proteins ${ }^{9-11}$. This molecular interaction leads to ubiquitin-dependent degradation of JAZ proteins, which in turn results in induced activity of the defence regulatory transcription factors (TFs) MYC2/MYC3/MYC4 (MYC2/3/4) and EIN3/EIL3 ${ }^{9,11-14}$. The MYC2/3/4 and EIN3/EIL1 branches of the JA response pathway are co-regulated by the plant hormones abscisic acid (ABA) and ethylene (ET), directing the JA pathway towards activation of defences against herbivory or necrotrophic pathogens, respectively $3,7,13,15-18$.

Compared to the short-term effects of JA, little is known about the long-term impacts of JA, despite the potential impacts transient stress responses can have on ecologically relevant life-history traits, such as growth rate, seed set and immune responsiveness ${ }^{1}$. It has been reported that treatment of Arabidopsis with methyl jasmonate (MeJA) elicits transgenerational IR against chewing herbivores ${ }^{19}$, suggesting an epigenetic basis of long- 
term JA-IR. However, the epigenetic mechanisms underpinning long-term JA-IR and their associated impacts on global gene expression remain poorly understood.

In plants, cytosine $(\mathrm{C})$ methylation occurs at three sequence contexts: $\mathrm{CG}, \mathrm{CHG}$ and $\mathrm{CHH}$ ( $\mathrm{H}$ being any base other than $\mathrm{G}$ ) and predominantly targets transposable elements (TEs) to silence their potentially damaging effects on the genome ${ }^{20}$. The establishment of methylation at TE-rich regions is under antagonistic control by RNA-directed DNA methylation $(\operatorname{RdDM})^{21,22}$ and the DNA demethylase ROS $1^{20,23}$. Over recent years, evidence has emerged that $\mathrm{RdDM}$ and ROS1 regulate plant defences against biotic stress ${ }^{24-26}$.

Here, we have investigated the long-term effects of JA on the defence-related phenotype, transcriptome and DNA methylome of Arabidopsis. We show that long-lasting JA-IR at 3 weeks after treatment of 2-week-old seedlings is effective against herbivory but not against pathogens. The response is associated with shifts in the defence-related transcriptome and metabolite profiles and is dependent on the MYC2/3/4 branch of the JA response pathway, RdDM- and ROS1-dependent DNA (de)methylation pathways, and the small RNA (sRNA)binding effector protein ARGONAUTE1 (AGO1). Global DNA methylome sequencing furthermore showed that long-term JA-IR is associated with highly specific hypomethylation of TEs from the ATREP2 family, while analysis of AGO1-associated sRNAs revealed increased association with sRNAs from this TE family. We propose a novel model of longlasting plant immune memory, involving ATREP2-derived small interfering RNAs (siRNAs) that augment MYC2/3/4-dependent resistance through nuclear AGO1. 


\section{RESULTS}

JA induces long-term resistance to a generalist herbivore and long-term susceptibility to both necrotrophic and hemi-biotrophic pathogens.

To examine the dynamics of the JA response over an extended time period, 2-week-old Arabidopsis seedlings were treated with water or $1 \mathrm{mM} \mathrm{JA}$ and analysed for JA-dependent MYC2 and VSP2 expression over a 3-week period (Fig. 1a,b). Both marker genes showed transient induction at 4 and 24 hours (hrs) post seedling treatment, after which their expression reverted to near baseline levels by 1 to 3 weeks (Fig. 1b). To assess the longterm effects of this transient JA signalling activity on the defence phenotype, we quantified resistance in 5-week-old plants, at 3 weeks after seedling treatment, against the generalist herbivore Spodoptera littoralis $(S I)$, the necrotrophic fungus Plectosphaerella cucumerina $(P c)$ and the biotrophic bacterial pathogen Pseudomonas syringae pv. tomato DC3000 (Pst; Fig. 1a). To compare these long-term effects with the short-term effects of JA, we also challenged an additional batch of 5-week-old plants with the same stresses at 1 day after treatment with water or $1 \mathrm{mM} \mathrm{JA} \mathrm{(Fig.} \mathrm{1a).} \mathrm{As} \mathrm{expected,} \mathrm{the} \mathrm{short-term} \mathrm{effects} \mathrm{of} \mathrm{JA} \mathrm{were}$ characterised by IR against both $S /$ and $P c$, as evidenced by a statistically significant reduction in larval weight and lesion diameter, respectively (Fig. 1c). Furthermore, JA treatment 1 day before Pst challenge increased bacterial leaf multiplication (Fig. 1c), supporting earlier reports that JA signalling suppresses SA-dependent resistance against biotrophic pathogens ${ }^{27,28}$. Interestingly, even though JA signalling activity had reverted to near basal levels at 1 week after JA seedling treatment (Fig. 1b), 5-week-old plants from JAtreated seedlings retained IR against SI and induced susceptibility (IS) to Pst (Fig. 1d). Whereas, in contrast to the short-term JA response, plants from JA-treated seedlings displayed IS to the necrotrophic fungus $P c$ (Fig. 1d), indicating a fundamental difference between the short- and long-term effects of JA on Arabidopsis immunity. To verify the biological relevance of these contrasting long-term effects of JA on SI and Pc resistance, we subjected seedlings to transient feeding by $S /$ larvae, which induces JA accumulation ${ }^{29}$. As 
observed after JA seedling treatment, seedling exposure to S/ feeding elicited long-term IR against SI and long-term IS to Pc (Extended Data Fig. 1). Hence, the long-term effects of transient JA signalling activity at the seedling stage are biologically relevant and phenotypically different to the short-term JA response.

\section{JA seedling treatment induces long-term priming of JA-dependent defences against} herbivory but represses SA- and JA/ET-dependent defences against pathogens.

Since MYC2 and VSP2 expression reverted to near basal levels by 3 weeks after JA seedling treatment (Fig. 1b), we hypothesised that long-term JA-IR against $S /$ is based on priming of JA-dependent defence genes. To test this, we quantified expression of MYCdependent anti-insect acid phosphatase gene VSP2 at 4, 8 and $24 \mathrm{hrs}$ after challenging leaves from seedling-treated plants with water or $0.1 \mathrm{mM} \mathrm{JA}$. Plants from JA-treated seedlings showed strongly augmented VSP2 induction after JA challenge, confirming that JA seedling treatment causes long-term priming of antiherbivore defences (Fig. 1e).

Conversely, plants from JA-treated seedlings showed reduced responsiveness of the SAinducible antimicrobial PR1 gene after challenge with $0.1 \mathrm{mM} \mathrm{SA}$, as well as the JA/ETdependent antifungal PDF1.2 gene after challenge with a mixture of $0.1 \mathrm{mM} \mathrm{JA}+0.1 \mathrm{mM}$ of the ethylene precursor 1-aminocyclopropanecarboxylic acid (ACC; Fig. 1e). Hence, JA seedling treatment induces long-lasting priming of JA-inducible VSP2 but long-term repression of SA-induced $P R 1$ and JA/ET-inducible PDF1.2.

\section{Long-term impacts of JA seedling treatment on the transcriptome.}

To assess the long-term impacts of JA on a global transcriptome level, we performed mRNA sequencing (mRNA-seq) of leaves from 5-week-old plants of water- and JA-treated seedlings at $4 \mathrm{hrs}$ after challenge with water or JA (Fig. 1e). Principal component analysis (PCA; Fig. 2a) and hierarchical cluster analysis (HCA; Fig. 2b) of normalised and transformed read counts revealed clear separation of samples by (pre)treatment $(n=4$; water seedling treatment and water challenge $=W_{-} \mathrm{W}, \mathrm{JA}$ seedling treatment and water challenge 
$128=\mathrm{JA} \_\mathrm{W}$, water seedling treatment and $\mathrm{JA}$ challenge $=\mathrm{W} \_\mathrm{JA}$ and $\mathrm{JA}$ seedling treatment and 129 JA challenge $=$ JA_JA). Hence, JA treatment of seedlings had a profoundly different impact 130 on the transcriptome than JA challenge treatment of 5-week-old plants.

131 Since JA seedling treatment altered the resistance/susceptibility to JA-eliciting attackers

132 (Fig. 1d), we hypothesised that JA seedling treatment modifies transcriptional responsiveness to secondary JA challenge. Accordingly, we selected genes showing a statistically significant interaction between JA seedling treatment and JA challenge (FDRadjusted $p$-value $<0.01$ ). The resulting 2,409 genes showed a range of different expression patterns (Extended Data Fig. 2 and Supplementary Data 1). To select for genes that are specifically associated with long-term JA-IR against SI, we filtered the 2,409 genes for those that (i) were upregulated upon JA challenge in plants from water-treated seedlings (W_JA > W_W) and (ii) showed augmented expression after JA challenge in plants from JA-treated seedlings compared to plants from water-treated seedlings (JA_JA > W_JA; Supplementary Data 2). HCA of the resulting 832 genes revealed four clusters, of which two (II and IV) displayed long-term upregulation and/or primed JA responsiveness in plants from JA-treated seedlings (Extended Data Fig. 3a and Fig. 2c). The 203 genes in clusters II and IV included the VSP2 marker gene and were statistically enriched with gene ontology (GO) terms related to herbivore resistance, particularly "glucosinolate biosynthetic process" (Extended Data Fig. 3b, Fig. 2d and Supplementary Data 3, 4 and 5). To select genes associated with long-term JA-IS to Pst, we filtered the 2,409 genes for those which (i) were downregulated in response to JA challenge in plants from water-treated seedlings $\left(W_{-} J A<W \_W\right)$ and (ii) showed reduced expression after JA challenge in plants from JA-treated seedlings compared to plants from water-treated seedlings (JA_JA $<$ W_JA; Supplementary Data 6). HCA of the resulting 904 genes revealed three clusters, of which two (V and VI) showed consistent short- and long-term repression by JA (Extended Data Fig. 3a and Fig. 2c). GO enrichment analysis of the 796 genes in clusters $\mathrm{V}$ and $\mathrm{VI}$ indicated enrichment of terms related to biotrophic pathogen resistance, including SA signalling (Extended Data Fig. 3b, Fig. 2d and 
155 Supplementary Data 7, 8 and 9). Finally, to select genes associated with long-term JA-IS to

$156 P c$, we filtered the 2,409 genes for those which (i) were upregulated in response to JA

157 challenge in plants from water-treated seedlings (

158 expression after JA challenge in plants from JA-treated seedlings compared to plants from

159 water-treated seedlings (JA_JA $<$ W_JA, Supplementary Data 10). HCA of the resulting 395

160 genes revealed one cluster (IX) with 144 genes showing long-term repression by JA and

161 significant enrichment with numerous GO terms related to necrotrophic pathogen resistance

162 (Extended Data Fig.3, Fig. 2c,d and Supplementary Data 11, 12 and 13). Thus, JA seedling

163 treatment induces long-term priming/upregulation of genes related to JA-dependent defence

164 against herbivores and long-term repression of SA- and ET-dependent genes against

165 biotrophic and necrotrophic pathogens.

Long-term JA-IR against herbivory is dependent on MYC2/3/4 transcription factors.

To further investigate the transcriptional regulation of long-term JA-IR against SI, we analysed the promoters of the 203 IR-related genes for statistical enrichment with TF DNA binding motifs (1 kb upstream from transcriptional start site; TSS). Most strongly enriched motifs contained the canonical G-box motif (CACGTG; Fig. 3a and Supplementary Data 14), which functions as a core binding site for bHLH TFs, including JA regulatory TFs MYC2/3/4 (Fig. 3a and Supplementary Data 14$)^{17,30,31}$. To validate involvement of MYC2/3/4 in longterm JA-IR against herbivory, we compared long- and short-term JA-IR against SI in 5-weekold Col-0 and the myc2 myc3 myc4 triple mutant $(m y c T)^{17}$. As reported previously ${ }^{17,32}$, watertreated $m y c T$ plants allowed significantly higher larval growth than water-treated Col-0 plants (Fig. 3b), reflecting their compromised basal resistance against herbivory. Furthermore, JAtreated Col-0 plants allowed significantly lower rates of larval growth than water-treated Col0 plants, confirming their ability to express short- and long-term JA-IR against $S$ (Fig. 3b). By contrast, JA treatment of mycT elicited neither short- nor long-term JA-IR against SI (Fig. $3 b)$, demonstrating a critical role of MYC2/3/4 TFs in both IR responses to this herbivore. 
182

treatment (Extended Data Fig. 4), indicating that long-term JA-IR is unrelated to JA-induced growth repression.

\section{Long-term JA-IR against herbivory requires intact DNA (de)methylation pathways.}

The defence-related phenotypes at 3 weeks after JA seedling treatment were expressed in leaves that were not present at the seedling stage, suggesting that there is a selfperpetuating resistance signal which is transmitted through cell division into the newly formed leaves. Changes in DNA methylation offer a plausible mechanism, since these can be transmitted through cell division ${ }^{33}$. Furthermore, previous studies have indicated that changes in DNA methylation of TEs controls defence gene expression ${ }^{25,34}$. Since TE methylation in Arabidopsis is controlled by the antagonistic activities of RdDM and the DNA demethylase ROS $1^{20}$, we investigated whether this regulatory system is required for JA-IR by testing two previously characterised mutants in RdDM (nrpe1-11) and ROS1 (ros1-4) ${ }^{25}$ for short- and long-term JA-IR against SI. Both mutants expressed similar levels of basal resistance and short-term JA-IR as the wild-type (Col-0; Fig. 4a). By contrast, long-term JAIR was strongly reduced in nrpe1-11 and ros 1-4 compared to Col-0 and failed to cause a statistically significant reduction in larval development (Fig. 4a). All genotypes displayed similar reductions in plant growth after JA seedling treatment, indicating that the lack of JAIR in nrpe1-11 and ros1-4 is unrelated to differences in JA-induced growth repression (Extended Data Fig. 4).

To obtain further evidence of the role of RdDM and ROS1 in long-term JA-IR against herbivory, we performed dual-choice assays to detect differences in attractiveness to $\mathrm{SI}$ larvae between water- and JA-treated plants. At $20 \mathrm{hrs}$ after release of the larvae in the choice arenas, a significantly higher number of S/ larvae preferred water-treated Col-0 plants over JA-treated Col-0 plants, demonstrating that long-term JA-IR reduces the attractiveness to SI (Fig. 4b). By contrast, nrpe1-11 and ros1-4 plants from water- and JA-treated seedlings attracted similar numbers of larvae (Fig. 4b), confirming that the ros1-4 and nrpe1-11 mutants are similarly affected in long-term JA-IR against herbivory. Since ROS 1 is positively 
controlled by RdDM via a DNA methylation monitoring sequence within its promoter ${ }^{20,35}$, we propose that the phenotypic similarity of ros $1-4$ and nrpe1-11 is caused by reduced ROS1 expression $^{25,35}$

\section{Long-term JA-IR is associated with ROS1-dependent changes in indole} glucosinolates.

The 203 genes associated with long-term JA-IR against S/ were statistically enriched with genes controlling the biosynthesis of glucosinolates (Fig. 2 and Supplementary Data 1 and 5). This suggests that the composition and size of the glucosinolate pool may be altered long-term following JA seedling treatment and contribute to the IR against SI. Furthermore, this alteration could be dependent on changes in DNA methylation. We therefore used high performance liquid chromatography coupled with triple quadrupole mass spectrometry (HPLC-QqQ) to profile changes in glucosinolate content between WT and ros 1-4 plants following JA seedling treatment. JA had long-term effects on glucosinolate composition (Fig. 4d,e and Extended Data Fig. 5), predominantly altering concentrations of indole glucosinolates (IGs). The main IG compound, glucobrassicin (I3M), as well as its downstream derivative neoglucobrassicin (NMOI3M), showed a statistically significant increase in WT plants upon JA seedling treatment (Fig. 4d,e). Whereas the IG 4methoxyglucobrassicin (4MOI3M) was statistically repressed by JA seedling treatment. Interestingly, these long-term changes in IG profiles were strongly attenuated (I3M and NMO13M) or absent (4MOI3M) in the ros1-4 mutant (Fig. 4e). Hence, ROS1-dependent DNA hypomethylation is not only essential for long-term JA-IR (Fig. 4b,c) but also controls the associated shifts in IG composition (Fig. 4d,e).

The methylome of long-term JA-IR is characterised by variable DNA hypomethylation at TEs.

To assess the long-term impacts of JA on global DNA methylation, biologically replicated leaf samples $(n=3)$ from 5 -week-old plants at 3 weeks after seedling treatment were 
analysed by whole-genome bisulfite sequencing (WGBS). For all sequence contexts the genome-wide weighted $\mathrm{C}$ methylation levels were comparable to previously reported values $^{36}$ (Extended Data Fig. 6a). Furthermore, although JA-treated samples showed on average marginally lower levels of genome-wide weighted $\mathrm{C}$ methylation, the differences were not statistically significant for any sequence context (Extended Data Fig. 6a). PCA and HCA of $\mathrm{C}$ methylation did not indicate consistent directional effects of JA seedling treatment. However both analyses revealed strongly increased variation in $\mathrm{C}$ methylation between replicate samples of JA-treated plants (Fig. 5a,b), which was driven by $\mathrm{CHG}$ and $\mathrm{CHH}$ sequence contexts (Fig. 5b and Extended Data Fig. 6b). Since non-CG methylation mostly occurs at intergenic TE sequences ${ }^{37}$, we hypothesised that the increased variation in DNA methylation in plants from JA-treated seedlings occurs at TEs. To test this hypothesis, we selected differentially methylated regions (DMRs) between each individual JA replicate and all 3 water replicates (1JA_vs_3W). In contrast to statistical comparisons between 3 replicates from each treatment (3JA_vs_3W), which selects for DMRs that are consistently different across replicate JA samples, the 1JA_vs_3W comparisons allows for identification of statistically significant DMRs that are variable between replicate JA samples. For DNA methylation at all sequence contexts (all-C), the three 1JA_vs_3W comparisons identified 325, 291 and 260 DMRs, respectively (Fig. 5c and Supplementary Data 15 and 16). Although these DMRs were relatively small (average $41 \mathrm{bp}$ ), they were C-rich (average 13 $\mathrm{C} / \mathrm{DMR}$ ) and showed substantial shifts in $\mathrm{C}$ methylation (average difference in methylation level of 43 percentage points; Supplementary Data 16). The 1JA_vs_3W comparisons for $\mathrm{CHH}$ context identified an average of 588 DMRs (Fig. 5c and Supplementary Data 15 and 16), while the comparisons for CHG and CG contexts yielded only 52 and 28 DMRs, respectively (Fig. 5c and Supplementary Data 15 and 16). These sequence-specific DMRs were also relatively small (average 53, 50 and $42 \mathrm{bp}$ for $\mathrm{CHH}, \mathrm{CHG}$ and $\mathrm{CG}$, respectively) with substantial changes in $C$ methylation (average difference of 45,37 and 41 percentage points for $\mathrm{CHH}, \mathrm{CHG}$ ad CG, respectively; Supplementary Data 16). Notably, the majority of DMRs across all contexts and comparisons overlapped with TEs at intergenic regions and 
were hypomethylated in plants from JA-treated seedlings (Fig. 5c and Supplementary Data 16). Hence, the long-term variation in DNA methylation by JA seedling treatment is largely driven by variable hypomethylation of TEs at non-CG context.

\section{Long-term JA-IR is not associated with cis-acting DMRs within promoters of differentially expressed defence genes.}

\section{DNA methylation in gene promoters can influence the binding of TFs to gene promoters} motifs $^{20,38}$, which supports previous studies that have linked changes in gene expression and/or responsiveness to differential DNA methylation of the corresponding promoters $^{24,25,35,39}$. Although our global WGBS analysis suggests that JA-induced changes in DNA methylation occur at variable locations (Fig. 5a,b and Extended Data Fig. 6b), we examined whether DMRs from the different 1JA_vs_3W comparisons cluster within wider consensus regions of the same promoter regions. To this end, we searched for regions encompassing three DMRs, one from each of the 1JA_vs_3W comparisons (for details, see Methods). At all-C context, we identified 2 consensus DMRs, which mapped to the same region on chromosome 1 and were not located at gene promoters (Supplementary Data 17). Increasing the maximum distance between individual DMRs from 100 to $500 \mathrm{bp}$ did not yield additional consensus DMRs (Supplementary Data 17). Furthermore, we did not identify consensus DMRs at CG or CHG contexts and only identified 10 and 25 consensus DMRs at $\mathrm{CHH}$ context, using 100 and 500 bp maximum gaps, respectively (Supplementary Data 17). Although 7 and 19 of the latter $\mathrm{CHH}$ consensus DMRs were located within gene promoters, including WRKY14 (AT1G30650), GAT1 (AT1G08230) and CAM7 (AT3G43810, Supplementary Data 17), none of these genes were differentially expressed in our transcriptome analysis (Supplementary Data 1). We therefore conclude that the regulatory role of RdDM and ROS1 in long-term JA-IR (Fig. 4) does not stem from cis-acting DMRs in promoters of MYC2/3/4-dependent defence genes. 
290 Recent evidence suggests that DNA hypomethylation of TEs can stimulate defence gene

291 expression via trans-regulatory mechanisms ${ }^{1,34,40}$, offering an alternative mechanism by

292 which RdDM- and ROS1-dependent methylation controls long-term JA-IR. Various transacting mechanisms have been proposed, including activities by TE-derived small interfering RNAs (siRNAs) ${ }^{1}$. In the case of long-term JA-IR, however, such trans-regulating siRNAs would unlikely be generated by the same set of hypomethylated TEs, since there were only a few consensus DMRs between plants from JA-treated seedlings (Supplementary Data 17).

297 Since TEs within the same family and/or related families are highly homologous ${ }^{41}$, we hypothesised that different TEs from the same taxonomic family can have similar transacting activities. To test this hypothesis, we first mined our data for TE (super)families that are significantly enriched with JA-induced DMRs. Strikingly, the Helitron TE family ATREP2 stood out with on average a 11-fold (all-C) and 8-fold $(\mathrm{CHH})$ enrichment with JA-induced DMRs compared to the genomic background of all TEs, which was highly significant for all 1JA_vs_3W comparisons at all-C and $\mathrm{CHH}$ contexts (Fig. 5d,e). These ATREP2 DMRs were mostly hypomethylated and spread evenly across all chromosomes (Extended Data Fig. 7), but none were part of consensus DMRs in the proximity of differentially expressed defence genes (Supplementary Data 1 and 17). Apart from ATREP2, there were a small number of additional TE families that were weakly enriched at JA-induced CHH DMRs (Fig. 5e and Extended Data Fig. 8a), but they did not show the same fold-enrichment and statistical significance as ATREP2, nor were they consistently enriched across all three 1JA_vs_3W all-C context comparisons (Fig. 5d,e and Extended Data Fig. 8a,b). For JA-induced DMRs at CG and CHG contexts, there was weak enrichment of the Gypsy superfamily of LTR retrotransposons (Extended Data Fig. 8c,d). However, this enrichment was borderline statistically significant and did not translate to enrichment of specific TE families (Extended Data Fig. 8c,d). Thus, despite the variation in DNA hypomethylation, JA seedling treatment consistently targets TEs from the ATREP2 family. Combined with the observed up-regulation and/or priming of MYC2/3/4-dependent defence genes (Figs. 2 and 3) and the critical role of RdDM- and ROS1-dependent DNA methylation in long-term JA-IR (Fig. 4), our WGBS 
results suggest that stochastic hypomethylation of members from the ATREP2 TE family induce and/or prime JA-dependent defence genes via trans-acting mechanisms.

AGO1 associates with siRNAs derived from ATREP2 TEs and is essential for longterm JA-IR against herbivory.

Recently, Liu and colleagues (2019) reported that AGO1 associates with sRNAs in the nucleus and stimulates JA-dependent defence gene expression by changing the genes' chromatin structure and recruiting Pol-II ${ }^{42}$. To examine whether sRNAs from hypomethylated ATREP2 TEs play a role in long-term JA-IR by associating with AGO1, we mined previous sequencing data of RNAs associated with AGO1 immunoprecipitated from nuclear extracts of MeJA-treated Arabidopsis ${ }^{42}$. To enrich for AGO1-associated siRNAs, RNA sequences from other known RNA classes were removed from the analysis. As is shown in Fig. 6a, ATREP2-derived sRNAs showed strongly increased association with AGO1 compared to sRNAs from two similarly sized class 2 families (ATREP7 - 164 members and TNAT1A - 162 members, respectively), which were not targeted for hypomethylation.

To confirm the function of AGO1 in long-term JA-IR, we quantified long-term JA-IR in two Arabidopsis lines carrying relatively weak mutant alleles of $A G O 1$ (ago1-45 and ago1-46) ${ }^{43}$, which were not majorly affected in growth and development. While the weight of SI larvae reared on WT plants from JA-treated seedlings was significantly reduced compared to larvae reared on naïve WT plants, this long-term JA-IR was absent in ago1-45 and ago1-46 plants (Fig. 6b). Hence, long-term JA-IR requires an intact AGO1 protein. Together, these results indicate that siRNAs from hypomethylated ATREP2 associate with nuclear AGO1 to prime and/or upregulate distant JA-dependent defence genes and mediate long-term JA-IR (Fig. $6 c)$. 


\section{Wilkinson et al}

\section{DISCUSSION}

The immediate signalling response to JA has been studied extensively ${ }^{44}$. As a result, the pathways controlling short-term JA-IR against herbivores and necrotrophic pathogens, as well as the antagonistic effects of JA signalling on SA-dependent resistance against biotrophic pathogens, are well-documented ${ }^{7,27,28}$. By contrast, the long-term impacts of JAdependent stress signalling have largely been overlooked, which does not do justice to the full breath of plant adaptive strategies. Our study shows that the long-term response to JA is phenotypically and mechanistically distinct from the short-term response (Fig. 1), involving changes in DNA methylation of TEs and the sRNA-binding protein AGO1 (Fig. 4-6).

IR is typically based on a combination of priming and prolonged upregulation of inducible defences $^{1}$. The 203 genes associated with long-term JA-IR showed long-term priming and/or prolonged upregulation after JA seedling treatment (Fig. 2c). Consistent with the IR phenotype against $S$, this gene set included genes with previously reported anti-herbivore activity (e.g.VSP1 and VSP2) ${ }^{45}$ and was statistically enriched with $\mathrm{GO}$ terms related to glucosinolate biosynthesis (Fig. 2d and Supplementary Data 4 and 5). Subsequent HPLCQqQ profiling of glucosinolates confirmed that plants expressing long-term JA-IR show significant changes in IG composition (Fig. 4d,e and Extended Data Fig. 5). Previous studies have demonstrated that these anti-herbivore defences are controlled by MYC2/3/4 TFs ${ }^{17,32}$. Indeed, enrichment analysis of TF DNA-binding motifs in promoters of the 203 IR-related genes revealed strong enrichment with MYC-binding G-box motifs (Fig. 3a) ${ }^{17,30}$, while the mycT mutant was impaired in long-term JA-IR against $S$ (Fig. 3b). Hence, the immunological memory of long-term JA-IR is retained at the MYC2/3/4-dependent branch of the JA pathway, resulting in priming and/or prolonged up-regulation of anti-herbivore genes.

Recent evidence points to an important role of DNA methylation in plant immunity ${ }^{24-26,34,40,46}$. In most studies, however, epigenetic resistance phenotypes are induced by artificial gene mutations affecting DNA methylation, which does not necessarily demonstrate biological relevance. By contrast, our study has shown that transient signalling activity by the plant's 
own stress hormone or caterpillar infestation induces immune memory against herbivory, which is transmitted and maintained in newly developed leaves and dependent on RdDM and the DNA demethylase ROS1 (Fig. 4). Since DNA methylation of TEs is tightly controlled by $\mathrm{RdDM}$ and $\mathrm{ROS} 1^{20,23}$, these results indicate that long-term JA-IR requires changes in the methylation status of TEs. Although RdDM and ROS1 have opposite effects on DNA methylation, their shared function in long-term JA-IR could be explained by the RdDMactivated DNA methylation monitoring sequence in the promoter of the $R O S 1$ gene $^{20,35}$. This 'methylstat' allows for negative feedback on excessive DNA methylation, ensuring tightly regulated homeostasis of TE activities. Our finding that long-term JA-IR is associated with genome-wide changes in non-CG methylation at TEs (Fig. 5c) supports the involvement of this regulatory system.

Biotic stress typically leads to genome-wide DNA hypomethylation in plants ${ }^{1,20,47}$. For instance, both Pst infection and SA treatment induce wide-spread hypomethylation in the genome of Arabidopsis ${ }^{48,49}$, while MeJA treatment has been reported to induce DNA demethylation in Brassica rapa ${ }^{50}$. In most cases, this stress-induced DNA hypomethylation is enriched at TE sequences ${ }^{1,47,48,51,52}$, which supports our finding that the vast majority of JAinduced occurred at TEs in non-CG context (Fig. 5c). However, in contrast to previous studies, our WGBS analysis revealed considerable variability in TE hypomethylation by JA. Furthermore, the few consensus DMRs in our dataset were not located near the MYC2/3/4dependent defence genes that were differentially expressed at 3 weeks after JA seedling treatment. Considering the critical role of RdDM and ROS1 in long-term JA-IR (Fig. 4), we propose that a variable pool of hypomethylated TEs can trans-stimulate the expression and/or responsiveness of MYC2/3/4-dependent defence genes to mediate long-term JA-IR (Figs. 2c,d and 3). This notion is supported by independent studies suggesting that hypomethylated TEs trans-regulate the expression and/or responsiveness of defence genes $^{1,25,34,40}$. 
Different mechanisms have been proposed for trans-regulation of defence genes by hypomethylated TEs, including changes in long-range heterochromatic interactions and activities by TE-derived siRNAs ${ }^{1,46}$. Evidence in support of the latter mechanism comes from the recent discovery that siRNA-associated AGO1 stimulates JA-dependent defence gene expression through interaction with the SWI/SNF chromatin-remodelling complex and recruitment of Pol- $\mathrm{II}^{42}$. Interestingly, two independent mutations in AGO1 blocked long-term JA-IR against $S I$ (Fig. 6b). In addition, we found that long-term JA-IR is specifically associated with hypomethylated DMRs at TEs of the ATREP2 family (Fig. $5 \mathrm{~d}$ ), which generate siRNA sequences that show increased association with nuclear AGO1 (Fig. 6a). Together, these results provide plausible evidence that prolonged up-regulation and priming of defence genes during long-term JA-IR is controlled by AGO1-associated siRNAs from hypomethylated ATREP2 TEs (Fig. 6c). As homologous members of the same TE family can generate similar siRNAs, this model also explains how variable patterns of DNA hypomethylation result in the same IR phenotype. Accordingly, our study has uncovered a novel mode of epigenetic stress memory in plants. 


\section{METHODS}

Plant materials and growth conditions.

411

412

413

414

415

416

417

418

419

420

421

422

423

424

425

426

427

428

All Arabidopsis genotypes used in this study are in the genetic background of the accession Columbia (Col-0). The ros1-4 (SALK_135293), ago1-45 (NASC ID = N67861) and ago1-46 (NASC ID = N67862) mutants were obtained from the Nottingham Arabidopsis Stock Centre (NASC) and the nrpe1-11 (SALK_029919) mutant was kindly provided by Professor Pablo Vera (Instituto de Biología Molecular y Celular de Plantas, Spanish National Research Council, Spain). Seeds of ros 1-4 and nrpe1-11 came from stocks that had previously been confirmed to carry the correct T-DNA insertions and display transcriptional knock-down of ROS1 and NRPE1 genes, respectively ${ }^{25}$. ago1-45 and ago1-46 seed stocks were confirmed to be the correct genotype using previously described derived cleaved amplified polymorphic sequences (dCAPS) assays ${ }^{43}$. The myc2 myc3 myc4 triple mutant $(m y c T)^{17}$ was kindly provided by Professor Roberto Solano (Centro Nacional de Biotecnología, Consejo Superior de Investigaciones Científicas (CNB-CSIC), Spain). Seeds were stored at $4{ }^{\circ} \mathrm{C}$ in the dark and suspended in deionised $\mathrm{H}_{2} \mathrm{O}\left(\mathrm{dH}_{2} \mathrm{O}\right)$ for 4 days to break dormancy, after which they were sown onto soil consisting of Scott's Levington M3 compost (Everris) and sand in a 3:1 ratio and cultivated under the following conditions: $8.5: 15.5 \mathrm{hr}$ day:night, $21^{\circ} \mathrm{C}, 45-70 \%$ relative humidity $(\mathrm{RH})$ and $100-250 \mu \mathrm{E} \mathrm{m} \mathrm{m}^{-2} \mathrm{~s}^{-1}$.

\section{Pathogen strains and cultivation.}

Plectosphaerella cucumerina strain $B M M(P C)$ was kindly provided by Professor Brigitte Mauch-Mani (University of Neuchâtel, Switzerland). Pc was continuously cultured on potato dextrose agar (PDA) in the dark and at $15-25^{\circ} \mathrm{C}$. Four weeks prior to spore collection, a plug of PC PDA was transferred to a new plate. Pseudomonas syringae pv. tomato DC3000 IUxCDABE (Pst) was kindly provided by Dr Jun Fan (John Innes Centre, UK) ${ }^{53}$. Glycerol stocks of $P$ st were stored at $-80^{\circ} \mathrm{C}$. Two days prior to inoculation, a glycerol stock was 
434 thawed on ice and then cultured at $28{ }^{\circ} \mathrm{C}$ on King's $B(K B)$ agar plates supplemented with

435 rifampicin $\left(50 \mu \mathrm{g} \mathrm{ml}^{-1}\right)$ and kanamycin $\left(50 \mu \mathrm{g} \mathrm{ml}^{-1}\right)$.

$436 \quad$ Insect rearing.

437 Spodoptera littoralis (SI) eggs were kindly provided by Professor Ted Turlings (University of

438 Neuchatel, Switzerland). Larvae were reared in-house on a semi-artificial diet, which was

439 formulated based on the diets in refs. ${ }^{54-56}$. A full diet ingredient list is provided in

440 Supplementary Table 1 . The diet was prepared by autoclaving the agar in half the volume of 441 water $(300 \mathrm{ml})$ and then mixing with the additional ingredients.

\section{Chemical treatments.}

443 Stock solutions were prepared by diluting jasmonic acid (JA; Sigma Aldrich, J2500), 1aminocyclopropanecarboxylic acid (ACC; Sigma Aldrich, A3903) and salicylic acid (SA; Sigma Aldrich, S3007) in absolute ethanol (JA and SA; Fisher Scientific, E/0650DF/17) or $\mathrm{dH}_{2} \mathrm{O}(\mathrm{ACC})$. Solutions for plant treatments were prepared by diluting stocks with $\mathrm{dH}_{2} \mathrm{O}$ and supplementing with $0.02 \%$ of the surfactant silwet L-77 (LEHLE SEEDS, VIS-30). Pretreatment was performed with $1 \mathrm{mM} \mathrm{JA}$. Challenge consisted of $0.5 \mathrm{mM} \mathrm{SA}, 0.1 \mathrm{mM} \mathrm{JA}$ or $0.1 \mathrm{mM} \mathrm{JA}+0.1 \mathrm{mM}$ ACC. The controls for both the pre-treatment ('control') and challenge ('mock') consisted of $\mathrm{dH}_{2} \mathrm{O}$ supplemented with the same percentage ethanol as in the corresponding hormone solution. Pre-treatments were performed with either 2-week-old seedlings (long-term experiments; 3 weeks prior to challenge) or nearly 5 -week-old plants (short-term experiments; 1 day prior to challenge). Challenge treatments were performed when plants were 5 weeks old. All chemical treatments were performed by spraying plants until the leaf surfaces were entirely covered by liquid.

\section{Seedling treatment by herbivory.}

457 To test the long-term effects of transient seedling exposure to herbivory, $2^{\text {nd }}$ instar $S /$ larvae were placed on 2-week-old Col-0 plants and allowed to feed until 50-75\% of above ground tissue had been removed. To prevent (lethal) damage to the hypocotyl and encourage larvae 
460 to feed from the cotyledons and leaves, soil was piled around the hypocotyl and a $15 \mathrm{ml}$

461 falcon tube was placed over each plant. These protective measures were also applied to 462 control plants without larvae.

\section{Quantification of IR against necrotrophic Pc.}

464 Four leaves of the same developmental stage on 5-week-old plants (17-22 plants per treatment-genotype combination) were droplet-inoculated with $6 \mu$ droplets of $P c$ inoculum $\left(5 \times 10^{6}\right.$ spores $\left./ \mathrm{ml} \mathrm{H}_{2} \mathrm{O}\right)$, as described previously ${ }^{57,58}$. Inoculated plants were maintained at $100 \% \mathrm{RH}$. Lesion diameters were measured at 6-8 days post inoculation (dpi) and averaged into a single value per plant (unit of biological replication).

\section{Quantification of IR against hemi-biotrophic Ps.}

Four leaves of the same developmental stage on 5-week-old plants (9-12 plants per treatment-genotype combination) were syringe-infiltrated with a $10 \mathrm{mM} \mathrm{MgSO}_{4}$ suspension containing Pst bacteria at $\mathrm{OD}_{600 \mathrm{~nm}}=0.0002^{59}$. Plants were maintained at $80-100 \% \mathrm{RH}$. At 3 $\mathrm{dpi}, 4$ leaf disks $\left(0.2 \mathrm{~cm}^{2}\right)$ were harvested per plant and pooled (unit of biological replication). Leaf discs were homogenised in $10 \mathrm{mM} \mathrm{MgSO}_{4}$ and 5 -fold dilution series were plated on $\mathrm{KB}$ agar plates supplemented with rifampicin $\left(50 \mu \mathrm{g} \mathrm{ml}^{-1}\right)$ and kanamycin $\left(50 \mu \mathrm{g} \mathrm{ml}^{-1}\right)$. Plates were incubated at $28{ }^{\circ} \mathrm{C}$ for $20 \mathrm{hrs}$ and $4{ }^{\circ} \mathrm{C}$ for $17 \mathrm{hrs}$ prior to colony counting. Colonisation was expressed as the number of colony forming units (cfu's) per $\mathrm{cm}^{2}$ of leaf tissue.

\section{Quantification of IR against SI.}

To quantify larval growth in no-choice assays, 5-week-old plants (15-24 plants per treatmentgenotype combination) were grown individually in $425 \mathrm{ml}$ transparent plastic cups with three $0.8 \mathrm{~cm}^{2}$ holes drilled in the bottom to allow for water drainage. A single $S /$ neonate larva was placed on each plant with a fine paintbrush and a transparent lid was placed on each cup. Larvae were removed and weighed when complete consumption of the most susceptible phenotype was imminent or after 7 days, whichever came first. The weight of a single larva fed on an individual plant represented the unit of biological replication. 
486 To quantify herbivore attractiveness in dual-choice assays, 5-week-old plants were placed in

487 the dual-choice arenas (18 per genotype) at 3 weeks after seedling treatment. Every arena

488 consisted of two plants from water- or JA-treated seedlings of the same genotype, which

489 were in separate pots positioned in a $1 \mathrm{~L}$ transparent plastic container backfilled with soil and 490 separated by a $30 \mathrm{~mm}$ inverted Petri dish lid (the 'arena'). Five $2^{\text {nd }}-3^{\text {rd }}$ instar S/ larvae were 491 placed into the arena, after which containers were closed with pin-pricked lids. After $20 \mathrm{hrs,}$ the position of each larva was recorded. If larvae were not on a plant or the soil immediately under it, they were recorded as not making a choice.

\section{Hyperspectral quantification of plant size.}

Five-week-old plants (22-24 per treatment-genotype combination) were imaged, using a PlantScreen HC 900 hyperspectral imaging system (Photon Systems Instruments), consisting of a push-broom scanner with a halogen lamp light source and complementary metal-oxide-semiconductor detector (spatial resolution $=1,000$ pixels and spectral resolution $=0.8 \mathrm{~nm}$ ) mounted on a motorised carriage, which travelled directly over trays of plants at $15 \mathrm{~mm} \mathrm{~s}^{-1}$. The camera lens was positioned $20 \mathrm{~cm}$ above the rosettes and a $0.09 \mathrm{~s}$ exposure time was used. Raw intensity values were acquired for 480 wavebands across a $350-900 \mathrm{~nm}$ spectral range.

Plant size was approximated based on rosette surface area (RSA), which was quantified as the number of pixels in an image associated with one plant (unit of biological replication). calibrated reflectance image $(R)$ was produced, with reflectance values for all wavebands and pixels being generated using the following equation:

509 The intensity values were taken from one raw hyperspectral image $\left(I_{\text {raw }}\right)$ and two reference 510 images of the same white Teflon standard, one of which was taken in the light $\left(I_{\text {light }}\right)$ and one 511 in complete darkness $\left(I_{\text {dark }}\right)$. (ii) The wider area of the calibrated image containing the plant of 
512 interest was defined. (iii) All pixels within the defined area with a plant index (equation 2) >

$513 \quad 0.53$ were selected.

(2) $\quad$ Plant index $=1.2\left(2.5\left(R_{740}-R_{672}\right)-1.3\left(R_{740}-R_{556}\right)\right)$

515

516

517

518

519

520

521

522

523

524

525

526

527

528

529

530

531

532

533

(iv) Approximately one layer of pixels was removed from the edge of each selection of plantassociated pixels ('plant mask'). Computational analyses of the hyperspectral photos were performed with PlantScreen Data Analyser software (Photon Systems Instruments) and R (v3.6.1).

\section{RNA extractions.}

Two-week-old WT plants were treated with either water (control) or JA. A subset of plants were challenged 3 weeks later with a water (mock) or chemical solution (JA, SA, or JA + ACC), as detailed above. Leaf material was harvested both before and at $4 \mathrm{hrs}, 24 \mathrm{hrs}, 1$ week and 3 weeks after seedling treatment (Fig. 1b) or at 4, 8 and 24 hrs after challenge treatment (Fig. 1e). For the seedling treatment only experiment (Fig. 1b), 2-6 similarly aged plants from the same tray ( $4 \mathrm{hrs}, 24 \mathrm{hrs}$ and 1 week) or 3-5 leaves of a similar developmental stage from a single plant ( 3 weeks), were pooled and used as units of biological replication $(n=2-3)$. For the seedling treatment + challenge experiments (Fig. 1e), 8 similarly aged leaves from 2 plants in the same tray were pooled and used as the units of biological replication $(n=2-4)$. Total RNA extractions were performed as described previously ${ }^{25,34}$.

\section{Reverse transcriptase-quantitative PCR (RT-qPCR).}

Genomic DNA removal and cDNA synthesis were performed as described previously ${ }^{25,34}$, using approximately $1 \mu \mathrm{g}$ of total RNA. The sample mixes were prepared with the RotorGene SYBR Green PCR Kit (Qiagen) and run in a Rotor-Gene Q (Qiagen) real-time PCR cycler. Reactions were run at the following cycling conditions: 1 cycle of 10 mins at $95^{\circ} \mathrm{C}$ and $35-40$ cycles of 10 seconds at $95^{\circ} \mathrm{C}$ and 40 seconds at $60^{\circ} \mathrm{C} . \mathrm{C}_{\mathrm{t}}$ values were based on 'take-off' values calculated by the Rotor-Gene $Q$ 2.3.5 software. $C_{t}$ values from reactions 
538 with primers against MYC2, VSP2, PDF1.2 and PR1 (Supplementary Table 2) were

539 calculated relative to a single calibrator sample, using real-time PCR efficiency values $(E+1)$

540 of each primer pair. For each sample, the resulting values were normalised to the average

541 values of 3 reference genes, GAPC2 (AT1G13440), UBC21 (AT5G25760) and MON1

542 (AT2G28390), and normalised against the mean relative expression values of replicates

543 harvested prior to seedling treatment (Fig. 1b) or at 4 hrs after mock challenge of water

544 seedling treated plants (Fig. 1e).

\section{Glucosinolate Profiling}

546 Leaf material for glucosinolate profiling was collected from 5-week-old WT and ros1-4 plants

547 pre-treated with water (control) or JA (1 mM) as 2-week-old seedlings. Biologically replicated

548 samples $(n=8)$ consisted of 8 leaves of similar age collected from 2 plants (4 leaves/plant).

549 Leaf tissue was flash frozen and then lyophilized. Extraction and quantification of

550 glucosinolates was performed as described previously ${ }^{60}$. Briefly, $5 \mathrm{mg}$ of dried tissue was

551 ground to a fine powder. Glucosinolates were extracted by addition of $1 \mathrm{ml}$ of $70 \%(\mathrm{v} / \mathrm{v})$

552 methanol/water solution to the powder, vortexed, heated (5 $\mathrm{min})$, shaken (15 $\mathrm{min})$,

553 centrifuged ( $5 \mathrm{~min}$ at $15000 \mathrm{~g}$ ), and the supernatant was transferred into new tubes. The

554 supernatant was diluted in $100 \%$ Milli-Q water, filtered through a $0.22-\mu \mathrm{m} \mathrm{KX}$ syringe filter

555 (PTFE 13-mm diameter; Mikrolab), and injected into the LC-MS/MS system.

556 Samples were analysed in multiple reaction mode (MRM) on an Agilent 1260 Infinity HPLC

557 system (Santa Clara) connected to an AB Sciex 4500 triple-quadrupole trap (QqQ) mass

558 spectrometer (QTRAP/MS; AB Sciex), equipped with electrospray ionization (ESI) source in

559 negative ion mode. For each compound, two MRM-transitions, which showed the best

560 signal-to-noise ratios, were monitored.

561 Chromatographic separation for glucosinolates was performed at $40{ }^{\circ} \mathrm{C}$ on a reversed-phase

562 Synergi Fusion-RP C18, 80A column (250 mm × $2 \mathrm{~mm}$ i.d., $4 \mu \mathrm{m}$; Phenomenex) equipped

563 with a Security Guard Cartridge (Phenomenex, KJ0-4282). A binary solvent mixture was 
564

565

566

567

568

569

570

571

572

573

574

575

576

577

578

579

580

581

582

583

584

585

586

587

588

589

590

used consisting of water (solvent A) and methanol (solvent B). Both solvents contained 20 $\mathrm{mM}$ acetic acid. The flow rate was $0.3 \mathrm{ml} / \mathrm{min}$, and the injection volume $20 \mu \mathrm{l}$. The binary gradient was set up as follows: 0-3 min, column equilibration (95\% A), 3-10 min, ramping to $(80 \% \mathrm{~A}), 10-17 \mathrm{~min}$, ramping to $(55 \% \mathrm{~A}), 17-35 \mathrm{~min}$, ramping to $(0 \% \mathrm{~A}), 35-38 \mathrm{~min}$, isocratic hold $(0 \% \mathrm{~A})$, 38-38.5 min, ramping back to $(95 \% \mathrm{~A})$, and $38.5-45 \mathrm{~min}$, column reequilibrating (95\% A). All data were collected using ABSciex Analyst software v1.6.2.

Quantitation was performed using ABSciex MultiQuant software v3.0.2. Samples were run in a randomized order.

\section{Statistical analysis of data from bioassays, hyperspectral imaging, glucosinolate} profiling and RT-qPCR.

All statistical analyses were performed in R v3.6.1. Data from $P S, P c$ and no-choice $S I$ assays, hyperspectral imaging, glucosinolate profiling and RT-qPCR experiments were analysed by linear models. If data showed normal distributions and homoscedasticity, the analysis was performed by two-sample t-tests (binary comparisons) or one-, two- or threeway ANOVAs followed by Tukey post-hoc tests (multiple groups). Welch two-sample t-tests were used when binary comparison data showed heteroscedasticity. If data showed heteroscedasticity and/or residuals did not follow a normal distribution, data were transformed (logged, squared, square-rooted or transformed with the Box-cox or YeoJohnson transformations). When transformations failed to yield normal distributions, data were analysed by non-parametric Mann-Whitney tests (binary comparisons) or KruskalWallis tests followed by Pairwise Wilcoxon Rank Sum Tests (multiple groups) with $p$-values adjusted using the FDR approach. In all cases, a difference was deemed statistically significant at $p<0.05$. To test for statistically significant changes in larval attractiveness in the dual-choice SI assays, total numbers of larvae choosing plants from water- or JA-treated seedings were analysed by a Goodness-of-fit test against the null hypothesis that larval numbers were equal across treatments $(p<0.05)$.

mRNA transcriptome analysis: library preparation and sequencing. 
591 mRNA-sequencing (mRNA-seq) analysis was based on the same total RNA extracts used

592 for RT-qPCR analysis of VSP2 expression at 4 hrs after water/JA challenge ( $n=4$; Fig. 1 d).

593 Quantity and quality of RNA was assessed using a Nanodrop and 2100 Bioanalyzer (Agilent 594 Technologies). All RNA extracts used for sequencing yielded RNA integrity numbers (RIN) of 595 at least 6.4. Library preparation and sequencing was performed by BGI Genomics. mRNAs 596 were isolated using an oligo dT-based selection for poly(A) tails. Sequencing was performed 597 with the BGISEQ-500 platform functioning in its single end mode. Across all 16 samples 598 598 million 50 bp single-end clean reads were generated, with an average of 37.4 million clean 599 reads per sample (Supplementary Data 18). On average $98.7 \%$ of nucleotides per sample 600 had a Phred quality score of $>20$ (Supplementary Data 18).

mRNA transcriptome analysis: read alignment and counting.

602

603

604

605

606

607

608

609

610

611

612

613

614

615

Read quality was assessed using FASTQC v0.11.5

(http://www.bioinformatics.babraham.ac.uk/projects/fastqc/) and MultiQC v1.761. The first 15 bases of reads were removed using the read trimming tool Trimmomatic v0.38 (options: 'SE', 'HEADCROP:15') ${ }^{62}$. Reads were aligned to the Arabidopsis genome (Ensembl Plants vTAIR10.40), using STAR v2.6.1b with default parameters ${ }^{63}$. All samples had read alignment efficiencies between 89.3-90.8\% (average 90.3\%; Supplementary Data 18). Numbers of reads mapping to each annotated gene were counted using HTSeq v0.9.1 (option: '-stranded $=$ no' $^{64}$.

mRNA transcriptome analysis: statistical analysis of the mRNA-seq data.

Read count tables were loaded into R v3.6.1 and genes with a total read count of $<100$ across all samples were removed. Read counts were normalised for library size and transformed with a variance stabilising transformation (VST) ${ }^{65}$. Principal component analysis (PCA) of the 16 samples was performed using the 'plotPCA' function from DEseq2 v1.24.066 and displayed with ggplot2 v3.2.1. The outcome of hierarchical cluster analysis (HCA) of the 
61616 samples was displayed using pheatmap v1.0.12, with the complete linkage clustering

617 method and Euclidean distances.

618 To identify differentially expressed genes (DEGs) associated with long-term JA-induced 619 changes in resistance against JA-eliciting attackers, we used DESeq2 ${ }^{66}$ to select for 620 expression profiles with a statistically significant interaction between JA seedling treatment 621 and JA challenge treatment. A total of 2,409 DEGs were selected with an FDR-adjusted $p$ 622 value $(p . a d j)<0.01$ (Extended Data Fig. 2). These represented genes that responded 623 differently to JA challenge as a result of JA seedling treatment. The expression profiles were 624 projected in an clustered heatmap by the 'aheatmap' function of NMF v0.21.0, using Ward's 625 method and Pearson correlation distances. VST-transformed count data were projected in 626 the heatmap as per gene z-scores.

627 To identify DEGs associated with the long-term JA-IR against SI, genes were selected based on their expression profile across the four treatment combinations (W_W, JA_W, W_JA, JA_JA; first letters indicate seedling treatment and second letters challenge treatment). Selected genes had to (i) be upregulated in response to JA challenge in plants from watertreated seedlings (W_JA $>$ W_W ) and (ii) exhibit augmented expression after JA challenge in plants from JA-treated seedlings compared to plants from water-treated seedlings (JA_JA $>$ W_JA). A clustered heatmap displaying the resulting 832 genes was created using Ward's clustering method and Spearman distances (Extended Data Fig. 3a). Based on expression profiles and enrichment of gene ontology (GO) terms related to anti-herbivore defences (see below), clusters II and IV with a total of 203 genes were selected for further analysis. This final set of IR-related genes were projected in a clustered heatmap using Ward's clustering method and Pearson distances (Fig. 2c).

DEGs were selected as associated with long-term JA-IS against $P$ st if they (i) were downregulated in response to JA challenge in plants from water-treated seedlings (W_JA < W_W) and (ii) exhibited reduced expression after JA challenge in plants from JA-treated seedlings compared to plants from water-treated seedlings (JA_JA $<$ W_JA). The resulting 
643

904 genes were displayed in a clustered heatmap using Ward's clustering method and Pearson distances (Extended Data Fig. 3a). Based on expression profiles and enrichment of GO terms relating to anti-pathogen defences (see below), clusters $\mathrm{V}$ and $\mathrm{VI}$ with a total of 796 genes were selected and displayed in a clustered heatmap created as before (Fig. 2c).

DEGs were selected as being associated with long-term JA-IS against $P_{C}$ if they (i) were upregulated in response to JA challenge in plants from water-treated seedlings (W_JA > W_W) and (ii) showed reduced expression after JA challenge in plants from JA-treated seedlings compared to plants from water-treated seedlings (JA_JA $<$ W_JA). The resulting 395 genes were displayed in a clustered heatmap using the average clustering method and Spearman distances (Extended Data Fig. 3a). Based on expression profile and enrichment of GO terms relating to anti-pathogen defences (see below), cluster IX with a total of 144 genes was selected and displayed in a clustered heatmap created as before (Fig. 2c).

mRNA transcriptome analysis: statistical enrichment analyses of gene ontology terms and TF DNA-binding motifs.

GO term enrichment analysis was performed with R packages clusterProfiler v3.12.0 and org.At.tair.db v3.8.2. For analysis of single and multiple gene clusters, the clusterProfiler functions 'compareCluster' and 'enrichGO', respectively, were used with parameters: 'universe $=$ all genes with $\geq 100$ counts across all 16 samples', 'fun = "enrichGO"' ('compareCluster' only), 'OrgDb = 'org.At.tair.db", 'keyType = "TAIR"', 'ont = 'BP', 'minGSSize $=10$ ' and 'maxGSSize $=500$ '. Biological process GO terms with a p.adj $<0.05$ were classed as enriched. Fold enrichment plots of selected enriched defence-related GO terms were created using the $\mathrm{R}$ package ggplot2.

For the TF DNA-binding motif enrichment analysis, promoter sequences (TSS to $1 \mathrm{~kb}$ upstream) for all genes analysed by DESeq2 ( $\geq 100$ reads across all samples), were downloaded from TAIR v10. These promoter sequences, together with the 803 Arabidopsis TF DNA-binding motifs found in the MotifDb v1.26.0 R package and the functions 
669 'makePriors', 'PFMtoPWM' and 'makeBackground' from the PWMEnrich v4.20.0 R package, 670 were used to create background distributions of TF DNA-binding motifs. To determine which 671 of the 803 MotifDb Arabidopsis motifs were significantly overrepresented $(p<0.01)$ in the

672203 IR-related genes promoters relative to the background, the PWMEnrich functions 673 'motifEnrichment' (all parameters default apart from 'group.only = F') and 'groupReport' (all 674 options default) were used. Sequence logos were produced using the PWMEnrich 'plot' 675 function.

Methylome analysis: library preparation and sequencing.

Leaf material for whole-genome bisulphite sequencing (WGBS) analysis was collected from 5-week-old plants that had been treated with water (control) or JA as 2-week-old seedlings. Biologically replicated samples $(n=3)$ consisted of 12 leaves of similar age collected from 6 plants per tray (2 leaves/plant). Genomic DNA was extracted using the GenElute Plant Genomic DNA Miniprep Kit (Sigma-Aldrich). Library preparation and sequencing was performed by BGI Genomics and their standard WGBS protocol. The sequencing of $150 \mathrm{bp}$ paired-end reads was performed with a HiSeq X Ten System (Illumina). Across all 6 samples, 97 million clean paired-end reads were generated, with a minimum and maximum number of 15.6 and 17 million read pairs per sample, respectively (Supplementary Data 19). On average, $98.2 \%$ of all nucleotides yielded a Phred quality score of $>20$ (Supplementary Data 19).

Methylome analysis: read alignment.

Read quality was assessed using FASTQC and MultiQC ${ }^{61}$. The first 10 bases were removed from the start of each read with Trimmomatic (options: 'PE', 'HEADCROP:10') ${ }^{62}$. Reads were aligned to the Arabidopsis genome (Ensembl Plants vTAIR10.40) using bismark v0.21.067, run with the default parameter settings which includes the use of Bowtie2 v2.3.4.168 for read mapping. Alignment efficiency for each of the 6 samples was between $58-66 \%$

694 (Supplementary Data 19). To remove duplicate reads, BAM alignment files were rearranged 
695

696

697

698

699

700

701

702

703

704

705

706

707

708

709

710

711

712

713

714

715

716

717

718

719

720

using SAMtools v1.7 (options: 'sort', '-n') ${ }^{69}$ and then passed to the Bismark tool 'deduplicate_bismark' (option: '--paired'). Between 23-29\% of aligned paired-end reads were removed from each sample in the deduplication procedure. After alignment and deduplication, between $43-51 \%$ of all sequenced paired-end reads were retained per sample (Supplementary Data 19).

Methylome analysis: methylation calling and determining weighted methylation levels.

Methylated and total (methylated + unmethylated) read counts per cytosine (C) position were generated using the Bismark tool 'bismark_methylation_extractor' (options: '--paired-end', '-no_overlap', ‘--ignore_3prime_r2 90', ‘--comprehensive', ‘--bedGraph', ‘--CX', ‘-cytosine_report'). Per sample bisulfite treatment non-conversion rates were estimated from the unmethylated plastid genome and ranged between $0.37-0.48 \%$ across all 6 samples (Supplementary Data 19 ; non-conversion rate $<2 \%$ is considered acceptable ${ }^{70}$ ). Counts for all $\mathrm{C}$ positions in the nuclear genome were used for downstream analysis of genome-wide methylation at all sequence contexts (all-C), as well as for $\mathrm{CG}, \mathrm{CHG}$ and $\mathrm{CHH}$ contexts separately ( $\mathrm{H}$ indicates any base other than $\mathrm{G})$. Estimates of genome-wide methylation levels were calculated using the weighted methylation level equation in ref. ${ }^{71}$.

\section{Methylome analysis: global analysis of positional cytosine methylation.}

To detect global shifts in DNA methylation, HCAs and PCAs were conducted for each of the 4 sequence contexts (all-C, CG, CHG and $\mathrm{CHH}$ ). Both analyses were performed with positional C-methylation data calculated using the site methylation level equation in ref. ${ }^{71}$. All C positions with a coverage $<5$ in one or more samples were removed. In addition, positions with a standard deviation of methylation lower than or equal to the median of the standard deviations of all cytosines across the whole genome were removed, thereby focusing the analyses on the most variable positions. PCAs were conducted with the R function 'prcomp' (options: 'center = TRUE', 'scale = FALSE'). HCA was performed with the R functions 'dist' and 'hclust' run with the options 'method = "euclidean"' and 'method = "average"', 
respectively. PCA and HCA plots were created with the R packages ggplot2 v3.6.1 and dendextend v1.13.4.

\section{Methylome analysis: analysis of differentially methylated regions.}

Since the global methylome analyses revealed increased variation in $\mathrm{C}$ methylation between replicate samples from plants of JA-treated seedlings, we adjusted our strategy for statistical selection of differentially methylated regions (DMRs) by selecting for DMRs that were statistically different between each individual sample from JA-treated plants and all three replicate samples from water-treated plants (1JA_vs_3W). This approach is not confounded by the increased variability between JA samples. To identify DMRs in each of the three all-C context 1JA_vs_3W comparisons, we used the DSS v2.26.0 R package functions 'DMLtest' (options: 'equal.disp = TRUE', 'smoothing = FALSE') followed by 'callDMR' (options: 'delta = 0.1 ', 'p.threshold $=0.05$ ', 'minlen $=25$ ', 'minCG $=5$ ', 'dis.merge $=50$ ', 'pct.sig $\left.=0.5^{\prime}\right)^{72,73}$ ' Since DSS accounts for coverage depth information, we included all C positions. Contextspecific DMRs were identified by running the same DSS analysis pipeline with $C$ positions at CG, $\mathrm{CHG}$ or $\mathrm{CHH}$ contexts only.

To map DMRs to genomic features, Arabidopsis genome and TE annotation files were downloaded from Ensembl vTAIR10.40 and TAIR v10, respectively. Analysis of DMRs overlapping with specific genomic features was conducted with the $\mathrm{R}$ packages GenomicRanges v1.36.1 and genomation v1.16.0. The precedence order for DMRs overlapping with genomic features was promotor $>$ exon $>$ intron $>$ intergenic. Statistical enrichment of TE (super)families within DMRs was determined by hypergeometric tests, using all TEs annotated in TAIR v10 as the background (p.adj<0.05). Plots of DMR frequencies and TE (super)family enrichments were created using the R packages ggplot2 and ggrepel v0.8.1. A chromosome map displaying the distribution of DMR-overlapped ATREP2 TEs was generated using the TAIR v10 gaps track downloaded from the UCSC genome browser, the centromere coordinates obtained from the TAIR v9 genome assembly and the R package chromPlot v1.12.0. 
748

749

750

751

752

753

754

755

756

757

758

759

760

761

762

763

764

765

766

767

768

769

770

771

772

773

774

Consensus DMRs were defined as wider regions encompassing one DMR from each of the three 1JA_vs_3W comparisons, and were selected using the following pipeline: (i) identified 'overlapping' DMRs from a pair of 1JA_vs_3W comparisons, using the 'findOverlaps' function from the R package GenomicRanges, (ii) created merged DMRs using the highest and lowest coordinates from across the DMR pair, (iii) identified DMRs from the third 1JA_vs_3W comparison which "overlapped" merged DMRs, using the 'findOverlaps' function, (iv) created consensus DMRs using the highest and lowest coordinates from across the three DMRs, (v) repeated steps i to iv three times to cover each possible combination of 1JA_vs_3W comparisons, and (vi) removed consensus DMR duplicates. The consensus DMR identification pipeline was run twice for each of the 4 sequence contexts (all-C, CG, $\mathrm{CHG}$ and $\mathrm{CHH}$ ). In the first run, pairs of DMRs were classed as "overlapping" if they were within $100 \mathrm{bp}$ of one another and in the second run if they were within $500 \mathrm{bp}$ of one another.

\section{Analysis of sRNAs associated with nuclear AGO1}

Raw sequencing reads of AGO1-associated RNAs from 10-day-old Col-0 at $1 \mathrm{hr}$ after MeJA treatment were downloaded from the NCBI Sequence Read Archive (SRR5313816). For full details on the experimental design and sequencing see Liu et al. ${ }^{42}$.

Adaptors were trimmed from reads and low-quality reads were removed using Trim Galore v0.6.2 (options: '--quality 0', '--length 18', '--max_length 30', '--stringency 6', '--max_n 0'). Quality of the remaining reads was assessed using FASTQC and MultiQC ${ }^{61}$. Reads were mapped to the Arabidopsis genome (Ensembl Plants vTAIR10.40) using Bowtie v1.3.0 (options: '-v 0', '--all', ‘--best', '--sam', ‘--no-unal'), with all alignments with no mismatches being reported. To focus the analysis on siRNAs plausibly involved in the trans-regulation of JA-dependent defence genes by hypomethylated TEs, SAMtools $v 1.7^{69}$, bedtools $v 2.30 .0^{74}$ and Picard v2.24.2 were used to remove reads mapping to known classes of RNAs (rRNAs, rRNAs, snRNAs, snoRNAs and miRNAs). Subsequently the same tools were used to calculate the number and size of sRNAs mapping to TEs of the ATREP2, ATREP7 and 
Wilkinson et al

775 TNAT1A families. Coordinates of known classes of RNAs and TEs annotated in the

776 Arabidopsis genome were downloaded from TAIR v10. An sRNA size against frequency

777 distribution plot was created with the R package ggplot2. 
Wilkinson et al

\section{DATA AVAILABILITY}

779 The mRNA-seq and WGBS data discussed in this publication have been deposited in

780 NCBI's Gene Expression Omnibus and are accessible through GEO SuperSeries accession

781 number GSE163271 (https://www.ncbi.nlm.nih.gov/geo/query/acc.cgi?acc=GSE163271,

782 reviewer access token: odifuiiopdijxgx). The sRNA-seq data featured in this publication was

783 downloaded from the NCBI Sequence Read Archive (SRR5313816). 
784

785

786

787

788

789

790

791

792

793

794

795

796

797

798

799

800

801

802

803

804

805

806

807

\section{REFERENCES}

1. Wilkinson, S. W. et al. Surviving in a Hostile World: Plant Strategies to Resist Pests and Diseases. Annu. Rev. Phytopathol. 57, 505-529 (2019).

2. Irieda, H. et al. Conserved fungal effector suppresses PAMP-triggered immunity by targeting plant immune kinases. Proc. Natl. Acad. Sci. U. S. A. 116, 496-505 (2019).

3. Erb, M. \& Reymond, P. Molecular Interactions Between Plants and Insect Herbivores. Annu. Rev. Plant Biol. 70, 527-557 (2019).

4. Pel, M. J. C. \& Pieterse, C. M. J. Microbial recognition and evasion of host immunity. J. Exp. Bot. 64, 1237-1248 (2013).

5. Ahmad, S., Gordon-Weeks, R., Pickett, J. \& Ton, J. Natural variation in priming of basal resistance: From evolutionary origin to agricultural exploitation. Mol. Plant Pathol. 11, 817-827 (2010).

6. Bigeard, J., Colcombet, J. \& Hirt, H. Signaling mechanisms in pattern-triggered immunity (PTI). Mol. Plant 8, 521-539 (2015).

7. Pieterse, C. M. J., Van der Does, D., Zamioudis, C., Leon-Reyes, A. \& Van Wees, S. C. M. Hormonal modulation of plant immunity. Annu. Rev. Cell Dev. Biol. 28, 489-521 (2012).

8. Glazebrook, J. Contrasting mechanisms of defense against biotrophic and necrotrophic pathogens. Annu. Rev. Phytopathol. 43, 205-227 (2005).

9. Thines, B. et al. JAZ repressor proteins are targets of the SCFCOI1 complex during jasmonate signalling. Nature 448, 661-665 (2007).

10. Sheard, L. B. et al. Jasmonate perception by inositol-phosphate-potentiated COI1JAZ co-receptor. Nature 468, 400-407 (2010).

11. Howe, G. A., Major, I. T. \& Koo, A. J. Modularity in Jasmonate Signaling for 
Multistress Resilience. Annu. Rev. Plant Biol. 69, 387-415 (2018).

12. Chini, A., Gimenez-lbanez, S., Goossens, A. \& Solano, R. Redundancy and specificity in jasmonate signalling. Curr. Opin. Plant Biol. 33, 147-156 (2016).

13. Zhu, Z. et al. Derepression of ethylene-stabilized transcription factors (EIN3/EIL1) mediates jasmonate and ethylene signaling synergy in Arabidopsis. Proc. Natl. Acad. Sci. U. S. A. 108, 12539-12544 (2011).

14. Chini, A. et al. The JAZ family of repressors is the missing link in jasmonate signalling. Nature 448, 666-671 (2007).

15. Anderson, J. P. et al. Antagonistic Interaction between Abscisic Acid and JasmonateEthylene Signaling Pathways Modulates Defense Gene Expression and Disease Resistance in Arabidopsis. Plant Cell 16, 3460-3479 (2004).

16. Bodenhausen, N. \& Reymond, P. Signaling Pathways Controlling Induced Resistance to Insect Herbivores in Arabidopsis. Mol. Plant-Microbe Interact. MPMI 20, 1406-1420 (2007).

17. Fernández-Calvo, P. et al. The Arabidopsis bHLH transcription factors MYC3 and MYC4 are targets of JAZ repressors and act additively with MYC2 in the activation of jasmonate responses. Plant Cell 23, 701-715 (2011).

18. Song, S. et al. Interaction between MYC2 and ETHYLENE INSENSITIVE3 modulates antagonism between jasmonate and ethylene signaling in Arabidopsis. Plant Cell 26, 263-279 (2014).

19. Rasmann, S. et al. Herbivory in the previous generation primes plants for enhanced insect resistance. Plant Physiol. 158, 854-863 (2012).

20. Zhang, H., Lang, Z. \& Zhu, J. K. Dynamics and function of DNA methylation in plants. Nat. Rev. Mol. Cell Biol. 19, 489-506 (2018). 
21. Matzke, M. A. \& Mosher, R. A. RNA-directed DNA methylation: an epigenetic pathway of increasing complexity. Nat. Rev. Genet. 15, 394-408 (2014).

22. Cuerda-Gil, D. \& Slotkin, R. K. Non-canonical RNA-directed DNA methylation. Nat. Plants 2, 16163 (2016).

23. Tang, K., Lang, Z., Zhang, H. \& Zhu, J. The DNA demethylase ROS1 targets genomic regions with distinct chromatin modification. Nat. Plants 2, 16169 (2016).

24. $\mathrm{Yu}, \mathrm{A}$. et al. Dynamics and biological relevance of DNA demethylation in Arabidopsis antibacterial defense. Proc. Natl. Acad. Sci. U. S. A. 110, 2389-2394 (2013).

25. López Sánchez, A., Stassen, J. H. M., Furci, L., Smith, L. M. \& Ton, J. The role of DNA (de)methylation in immune responsiveness of Arabidopsis. Plant J. 88, 361-374 (2016).

26. Halter, T. et al. The Arabidopsis active demethylase ROS1 cis-regulates defence genes by erasing DNA methylation at promoter-regulatory regions. Elife 10, e62994 (2021).

27. Cui, J. et al. Pseudomonas syringae manipulates systemic plant defenses against pathogens and herbivores. Proc. Natl. Acad. Sci. U. S. A. 102, 1791-1796 (2005).

28. Murmu, J. et al. Arabidopsis GOLDEN2-LIKE (GLK) transcription factors activate jasmonic acid (JA)-dependent disease susceptibility to the biotrophic pathogen Hyaloperonospora arabidopsidis, as well as JA-independent plant immunity against the necrotrophic pathogen Botryti. Mol. Plant Pathol. 15, 174-184 (2014).

29. Scholz, S. S. et al. Mutation of the Arabidopsis calmodulin-like protein CML37 deregulates the jasmonate pathway and enhances susceptibility to herbivory. Mol. Plant 7, 1712-1726 (2014).

30. Lian, T. fei, Xu, Y. ping, Li, L. fen \& Su, X. D. Crystal Structure of Tetrameric Arabidopsis MYC2 Reveals the Mechanism of Enhanced Interaction with DNA. Cell 
Rep. 19, 1334-1342 (2017).

31. Carretero-Paulet, L. et al. Genome-wide classification and evolutionary analysis of the bHLH family of transcription factors in Arabidopsis, poplar, rice, moss, and algae. Plant Physiol. 153, 1398-1412 (2010).

32. Schweizer, F. et al. Arabidopsis basic helix-loop-helix transcription factors MYC2, MYC3, and MYC4 regulate glucosinolate biosynthesis, insect performance, and feeding behavior. Plant Cell 25, 3117-3132 (2013).

33. Law, J. A. \& Jacobsen, S. E. Establishing, maintaining and modifying DNA methylation patterns in plants and animals. Nat. Rev. Genet. 11, 204-220 (2010).

34. Furci, L. et al. Identification and characterisation of hypomethylated DNA loci controlling quantitative resistance in Arabidopsis. Elife 8, e40655 (2019).

35. Williams, B. P., Pignatta, D., Henikoff, S. \& Gehring, M. Methylation-Sensitive Expression of a DNA Demethylase Gene Serves As an Epigenetic Rheostat. PLoS Genet. 11, e1005142 (2015).

36. Niederhuth, C. E. et al. Widespread natural variation of DNA methylation within angiosperms. Genome Biol. 17, 194 (2016).

37. Cokus, S. J. et al. Shotgun bisulphite sequencing of the Arabidopsis genome reveals DNA methylation patterning. Nature 452, 215-219 (2008).

38. O'Malley, R. C. et al. Cistrome and Epicistrome Features Shape the Regulatory DNA Landscape. Cell 165, 1280-1292 (2016).

39. Gallego-Bartolomé, J. et al. Targeted DNA demethylation of the Arabidopsis genome using the human TET1 catalytic domain. Proc. Natl. Acad. Sci. U. S. A. 115, E2125E2134 (2018).

40. Cambiagno, D. A. et al. Immune receptor genes and pericentromeric transposons as 
targets of common epigenetic regulatory elements. Plant J. 96, 1178-1190 (2018).

41. Wicker, T. et al. A unified classification system for eukaryotic transposable elements. Nat. Rev. Genet. 8, 973-982 (2007).

42. Liu, C. et al. Arabidopsis ARGONAUTE 1 binds chromatin to promote gene transcription in response to hormones and stresses. Dev. Cell 44, 348-361 (2018).

43. Smith, M. R. et al. Cyclophilin 40 is required for microRNA activity in Arabidopsis. Proc. Natl. Acad. Sci. U. S. A. 106, 5424-5429 (2009).

44. Wasternack, C. How Jasmonates Earned their Laurels: Past and Present. J. Plant Growth Regul. 34, 761-794 (2015).

45. Liu, Y. et al. Arabidopsis vegetative storage protein is an anti-insect acid phosphatase. Plant Physiol. 139, 1545-1556 (2005).

46. Parker, A. A., Wilkinson, S. W. \& Ton, J. Epigenetics: a catalyst of plant immunity against pathogens. New Phytol. (2021).

47. Deleris, A., Halter, T. \& Navarro, L. DNA Methylation and Demethylation in Plant Immunity. Annu. Rev. Phytopathol. 54, 579-603 (2016).

48. Dowen, R. H. et al. Widespread dynamic DNA methylation in response to biotic stress. Proc. Natl. Acad. Sci. U. S. A. 109, E2183-E2191 (2012).

49. Pavet, V., Quintero, C., Cecchini, N. M., Rosa, A. L. \& Alvarez, M. E. Arabidopsis displays centromeric DNA hypomethylation and cytological alterations of heterochromatin upon attack by Pseudomonas syringae. Mol. Plant-Microbe Interact. 19, 577-587 (2006).

50. Kellenberger, R. T., Schlüter, P. M. \& Schiestl, F. P. Herbivore-Induced DNA demethylation changes floral signalling and attractiveness to pollinators in Brassica rapa. PLoS One 11, e0166646 (2016). 
905

51. Atighi, M. R., Verstraeten, B., De Meyer, T. \& Kyndt, T. Genome-wide DNA hypomethylation shapes nematode pattern-triggered immunity in plants. New Phytol. 227, 545-558 (2020).

52. Hewezi, T. et al. Cyst nematode parasitism induces dynamic changes in the root epigenome. Plant Physiol. 174, 405-420 (2017).

53. Fan, J., Crooks, C. \& Lamb, C. High-throughput quantitative luminescence assay of the growth in planta of Pseudomonas syringae chromosomally tagged with Photorhabdus luminescens luxCDABE. Plant J. 53, 393-399 (2008).

54. Gupta, G. P., Rani, S., Birah, A. \& Raghuraman, M. Improved artificial diet for mass rearing of the tobacco caterpillar, Spodoptera litura (Lepidoptera: Noctuidae). Int. J. Trop. Insect Sci. 25, 55-58 (2005).

55. Roeder, K. A., Kuriachan, I., Vinson, S. B. \& Behmer, S. T. Evaluation of a microbial inhibitor in artificial diets of a generalist caterpillar, Heliothis virescens. J. Insect Sci. 10, 197 (2010).

56. Bricchi, I. et al. Separation of early and late responses to herbivory in Arabidopsis by changing plasmodesmal function. Plant J. 73, 14-25 (2013).

57. Ton, J. \& Mauch-Mani, B. $\beta$-amino-butyric acid-induced resistance against necrotrophic pathogens is based on ABA-dependent priming for callose. Plant J. $\mathbf{3 8}$, 119-130 (2004).

58. Pétriacq, P., Stassen, J. \& Ton, J. Spore density determines infection strategy by the plant-pathogenic fungus Plectosphaerella cucumerina. Plant Physiol. 170, 2325-2339 (2016).

59. Yang, L. et al. Salicylic acid biosynthesis is enhanced and contributes to increased biotrophic pathogen resistance in Arabidopsis hybrids. Nat. Commun. 6, 7309 (2015).

60. Hooshmand, K. \& Fomsgaard, I. S. Analytical Methods for Quantification and 
Identification of Intact Glucosinolates in Arabidopsis Roots Using LC-QqQ(LIT)MS/MS. Metabolites 11, 47 (2021).

61. Ewels, P., Magnusson, M., Lundin, S. \& Käller, M. MultiQC: Summarize analysis results for multiple tools and samples in a single report. Bioinformatics 32, 3047-3048 (2016).

62. Bolger, A. M., Lohse, M. \& Usadel, B. Trimmomatic: a flexible trimmer for Illumina sequence data. Bioinformatics 30, 2114-2120 (2014).

63. Dobin, A. et al. STAR: ultrafast universal RNA-seq aligner. Bioinformatics 29, 15-21 (2013).

64. Anders, S., Pyl, P. T. \& Huber, W. HTSeq-A Python framework to work with highthroughput sequencing data. Bioinformatics 31, 166-169 (2015).

65. Anders, S. \& Huber, W. Differential expression analysis for sequence count data. Genome Biol. 11, R106 (2010).

66. Love, M. I., Huber, W. \& Anders, S. Moderated estimation of fold change and dispersion for RNA-seq data with DESeq2. Genome Biol. 15, 550 (2014).

67. Krueger, F. \& Andrews, S. R. Bismark: a flexible aligner and methylation caller for Bisulfite-Seq applications. Bioinformatics 27, 1571-1572 (2011).

68. Langmead, B. \& Salzberg, S. L. Fast gapped-read alignment with Bowtie 2. Nat. Methods 9, 357-359 (2012).

69. Li, H. et al. The Sequence Alignment/Map format and SAMtools. Bioinformatics 25, 2078-2079 (2009).

70. Stuart, T., Buckberry, S. \& Lister, R. Approaches for the Analysis and Interpretation of Whole Genome Bisulfite Sequencing Data. in Epigenome Editing: Methods and Protocols, Methods in Molecular Biology (eds. Jeltsch, A. \& Rots, M. G.) 299-310 
(Humana Press, 2018).

955

956

957

958

959

960

961

962

963

964

71. Schultz, M. D., Schmitz, R. J. \& Ecker, J. R. 'Leveling' the playing field for analyses of single-base resolution DNA methylomes. Trends Genet. 28, 583-585 (2012).

72. Feng, H., Conneely, K. N. \& Wu, H. A Bayesian hierarchical model to detect differentially methylated loci from single nucleotide resolution sequencing data. Nucleic Acids Res. 42, e69-e69 (2014).

73. $\mathrm{Wu}, \mathrm{H}$. et al. Detection of differentially methylated regions from whole-genome bisulfite sequencing data without replicates. Nucleic Acids Res. 43, e141 (2015).

74. Quinlan, A. R. \& Hall, I. M. BEDTools: A flexible suite of utilities for comparing genomic features. Bioinformatics 26, 841-842 (2010). 


\section{ACKNOWLEDGEMENTS}

966 We thank Professor Ted Turlings for providing Spodoptera littoralis eggs and Professor

967 Roberto Solano for providing the myc2 myc3 myc4 triple mutant. We also thank Leonardo

968 Furci, David Pascual-Pardo, Ellie Vinnicombe and David Rapley for assistance with

969 experiments and rearing of $S$. littoralis. The work presented in this publication was supported

970 by a consolidator grant (309944 "Prime-A-Plant") and a proof-of-concept grant (824985,

971 "ChemPrime") from the European Research Council to J.T., a Research Leadership Award

972 (RL-2012-042) from the Leverhulme Trust to JT, a BBSRC-IPA grant (BB/P006698/1) to

973 J.T., a PROMOS grant (Promo158) from the German Academic Exchange Service (DAAD)

974 and Freie Universität to A.M. and Research Council of Norway grants (249920 and

975 249958/F20) to P.K. and M.H.M., respectively. 
Wilkinson et al

976 AUTHOR CONTRIBUTIONS

977 S.W.W., P.K., M.H.M. and J.T. conceived the idea for the research, which was supervised by

978 J.T. S.W.W. conducted experiments and gathered data with assistance from R.S.W., A.M.,

979 M.A.H., A.L.S., E.K.M. and M.H.M. K.H. conducted the LC-MS/MS profiling of glucosinolates

980 with assistance from I.S.F. Data analysis was performed by S.W.W. with assistance from

981 R.S.W., A.M., M.A.H., E.K.M., J.H.M.S., A.L.S., K.H. and J.T. The paper was written by

982 S.W.W. and J.T. with comments and input from all other authors. M.H.M., P.K. and J.T.

983 provided funding for the research. 
Wilkinson et al

984 COMPETING INTERESTS STATEMENT

985 The authors declare no competing interests. 
$\therefore 8$

Sowing
Seedling treatment

(Long-term)
Treatment

(Short-term)

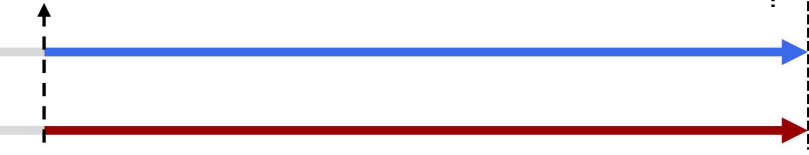

b

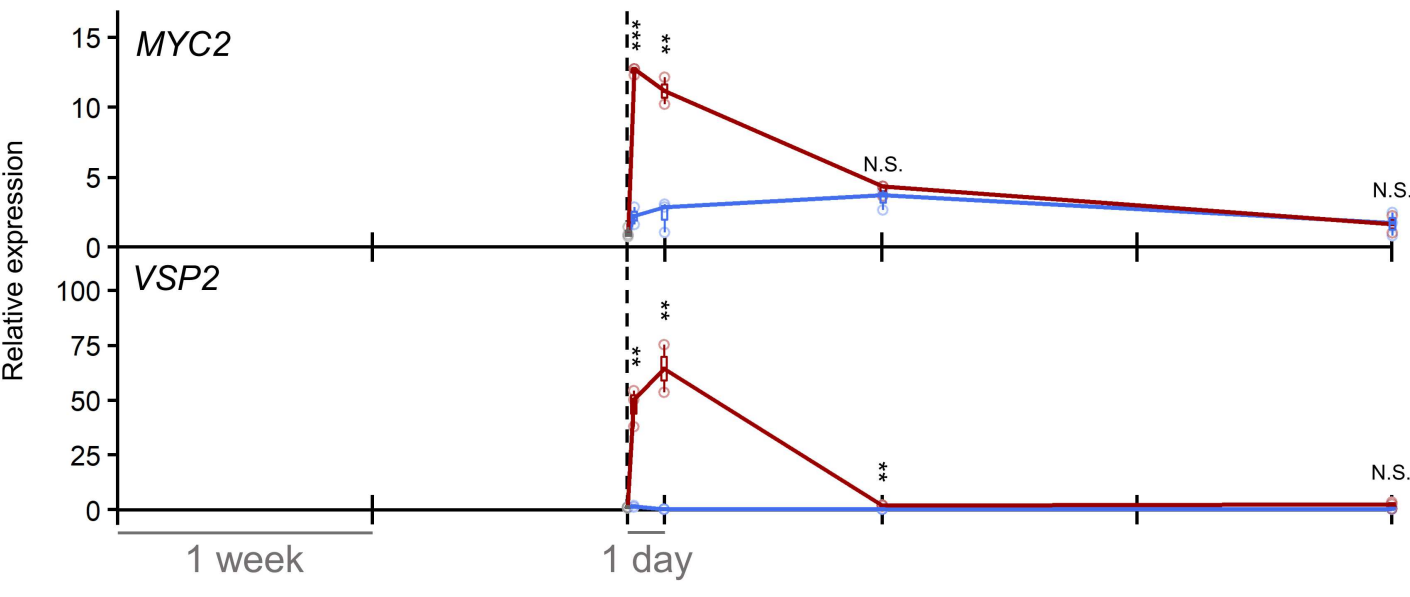

C

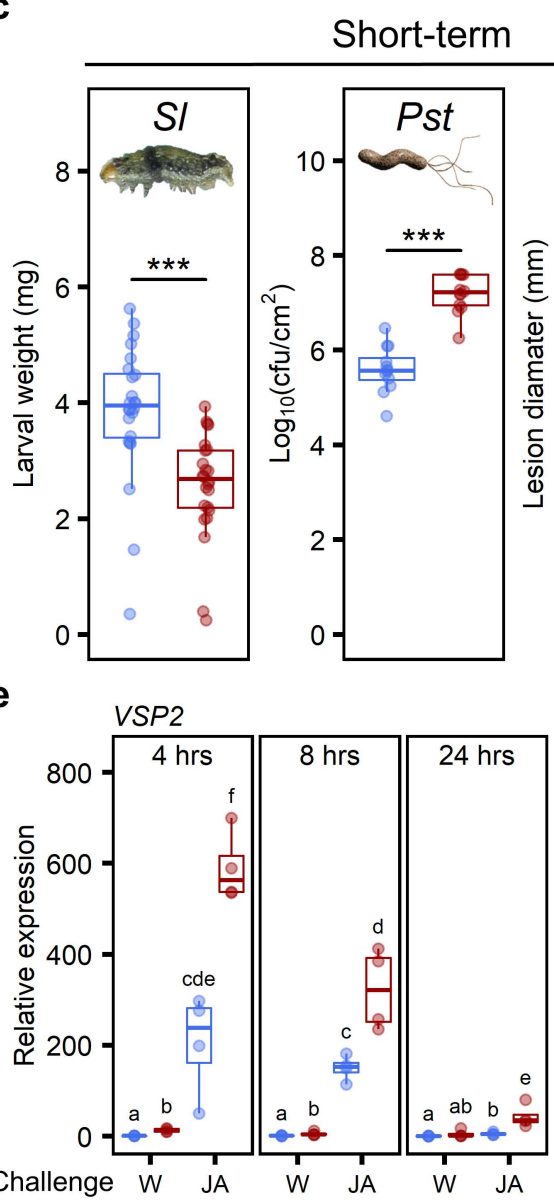

d

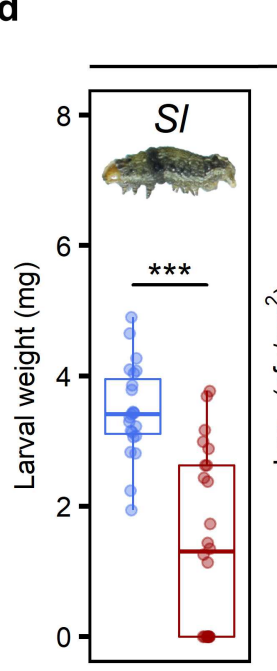

Long-term

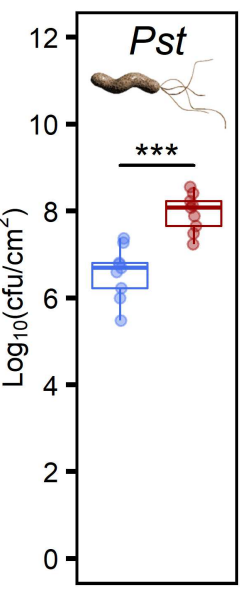

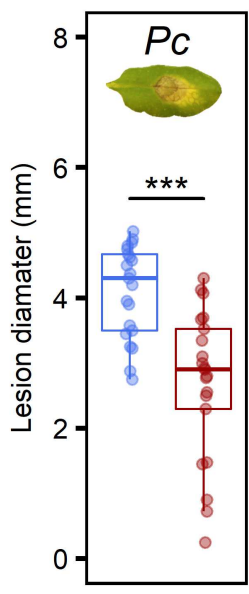

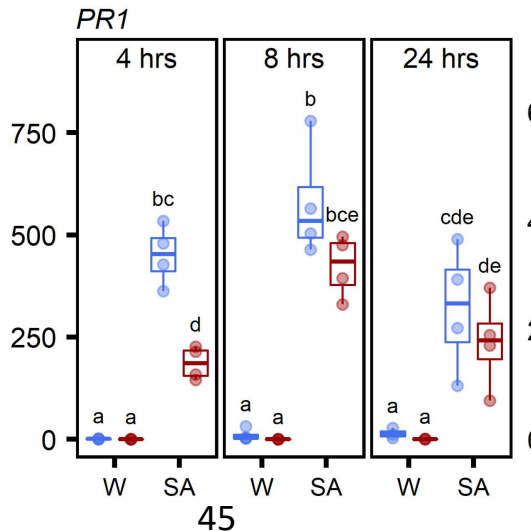

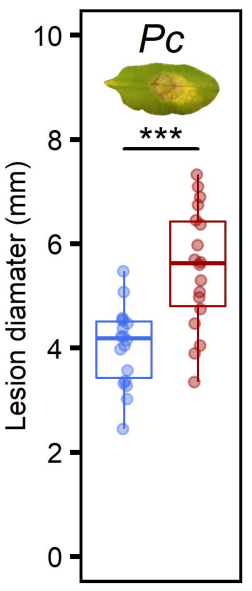

PDF1.2

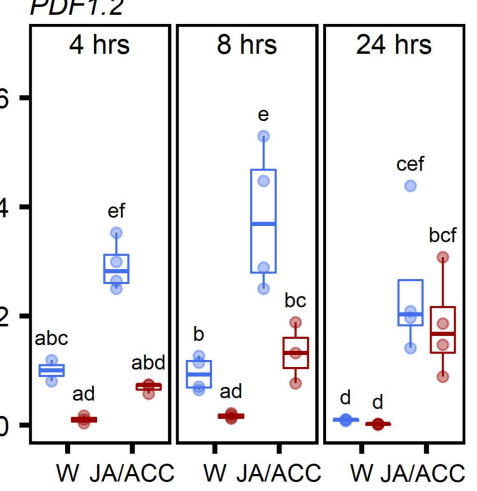


Fig. 1 | Short- and long-term effects of JA on resistance against three different biotic stresses. a, Experimental setup to analyse the short- and long-term impacts of jasmonic acid (JA) on biotic stress resistance in Arabidopsis (Col-0). Plants were pre-treated with water (control, blue) or $1 \mathrm{mM} \mathrm{JA} \mathrm{(red)} \mathrm{at} 1$ day (short-term) or 3 weeks (long-term) before stress challenge. $\mathbf{b}$, Long-term effects of seedling treatments on JA signalling activity over the 3-week period. Shown are the expression profiles (RT-qPCR) of the JA regulatory gene MYC2 and the JA marker gene VSP2. Data points represent gene expression values of biological replicates $(n=2-3)$ relative to the mean expression value of non-treated control plants at the time of seedling treatment (grey). Asterisks indicate statistically significant differences between treatments at individual time-points (Two-sample t-test; N.S. $p>0.05,{ }^{* *}$ $\left.p<0.01,{ }^{* * *} p<0.001\right) . \mathbf{c}, \mathbf{d}$, Short- and long-term effects of JA on resistance of 5-week-old plants against the herbivore Spodoptera littoralis $(S /)$, biotrophic pathogen Pseudomonas syringae pv. tomato DC3000 luxCDABE (Pst) and necrotrophic pathogen Plectosphaerella cucumerina $(P c)$. Data points represent weights of individual $S /$ larvae following feeding on individual plants ( $n=23-34$ ), mean colony forming units (cfu) of Pst per $\mathrm{cm}^{2}$ of leaf tissue per plant ( $n=9-12)$ and mean per plant lesion diameters by $P_{c}(n=18-21)$. Asterisks indicate statistically significant differences between pre-treatments (Two-sample t-test for $P c$ and $P s t$ assays, Welch two-sample t-test or Mann-Whitney test for S/ assays in $\mathbf{c}$ and $\mathbf{d}$, respectively; $\left.{ }^{* * *} p<0.001\right)$. e, Long-term effects of JA seedling treatment on the expression of defence marker genes upon challenge with either water (mock) or, $0.1 \mathrm{mM} \mathrm{JA}$ (VSP2), $0.5 \mathrm{mM}$ salicylic acid (SA; PR1) or $0.1 \mathrm{mM} \mathrm{JA} \mathrm{+} 0.1 \mathrm{mM}$ 1-aminocyclopropanecarboxylic acid (ACC; PDF1.2). Samples for RT-qPCR analysis were collected at 4, 8 and 24 hours (hrs) after challenge. Data points represent gene expression values of individual replicates $(n=2-4)$ relative to the mean expression values of control plants from water-treated seedlings at $4 \mathrm{hrs}$ post water challenge. Seedling treatment, challenge treatment and harvest timepoint combinations which do not share the same letter are significantly different (Kruskal-Wallis test followed by pairwise Wilcoxon rank sum tests for VSP2 or ANOVA followed by Tukey post-hoc test for PDF1.2 and PR1; p.adj < 0.05). Lower, middle and upper horizontal lines in 


\section{Wilkinson et al}

1015 boxplots indicate the $1^{\text {st }}, 2^{\text {nd }}$ and $3^{\text {rd }}$ quartiles; whiskers extend to the lowest and highest data 1016 points within $1.5 \times$ interquartile range below and above the $1^{\text {st }}$ and $3^{\text {rd }}$ quartiles. 
Wilkinson et al

a

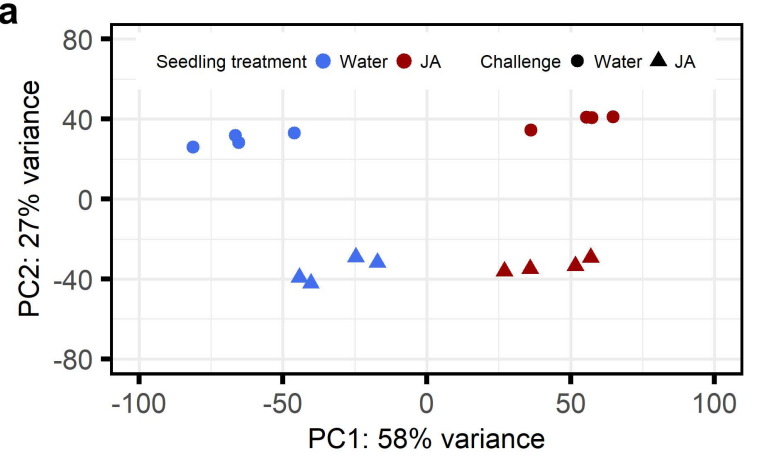

b

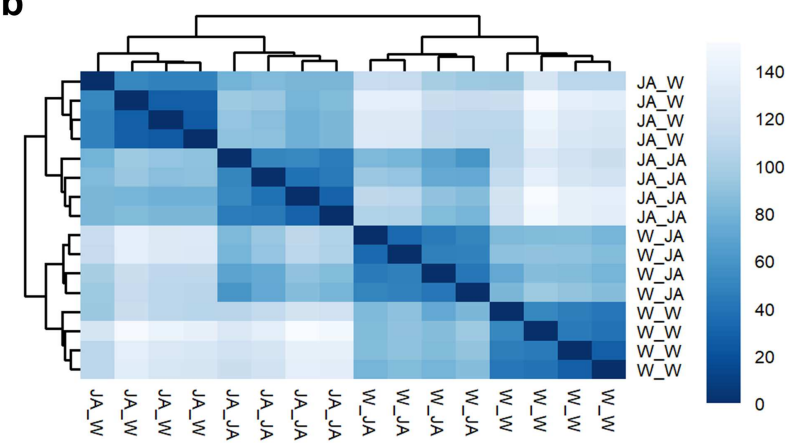

C

Gene profiles correlating with JA-IR to SI

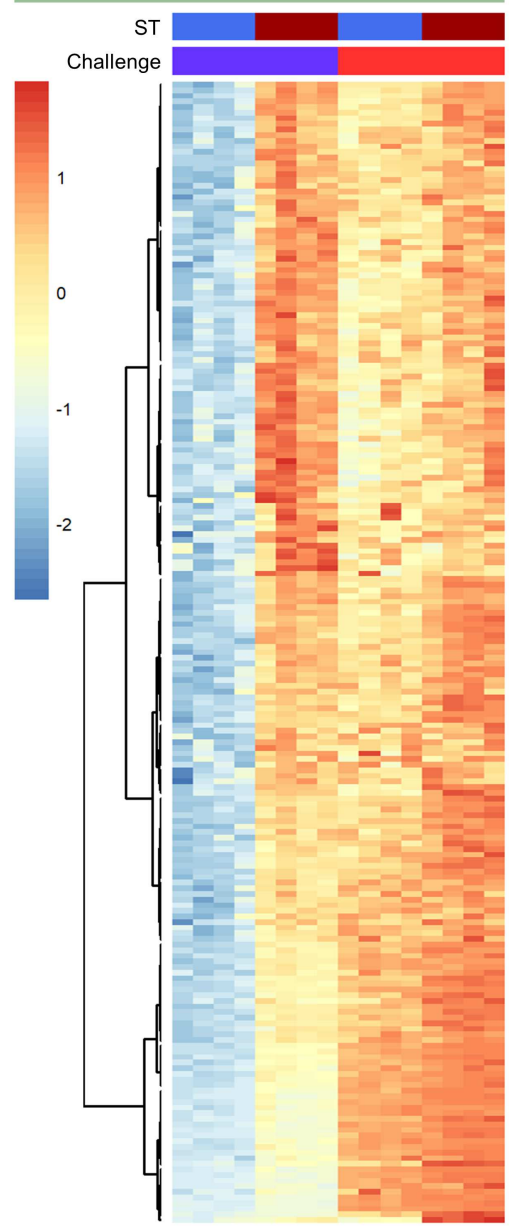

d

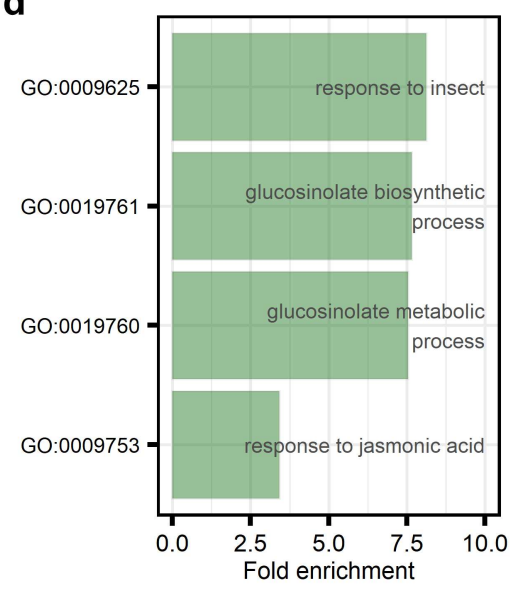

Gene profiles correlating with JA-IS to Pst

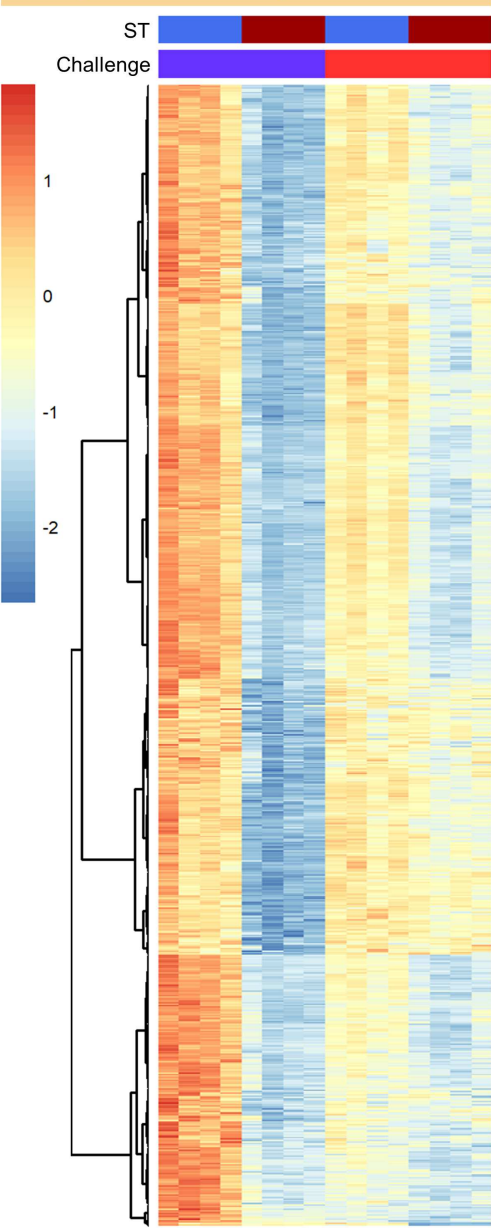

Gene profiles correlating with JA-IS to $P C$

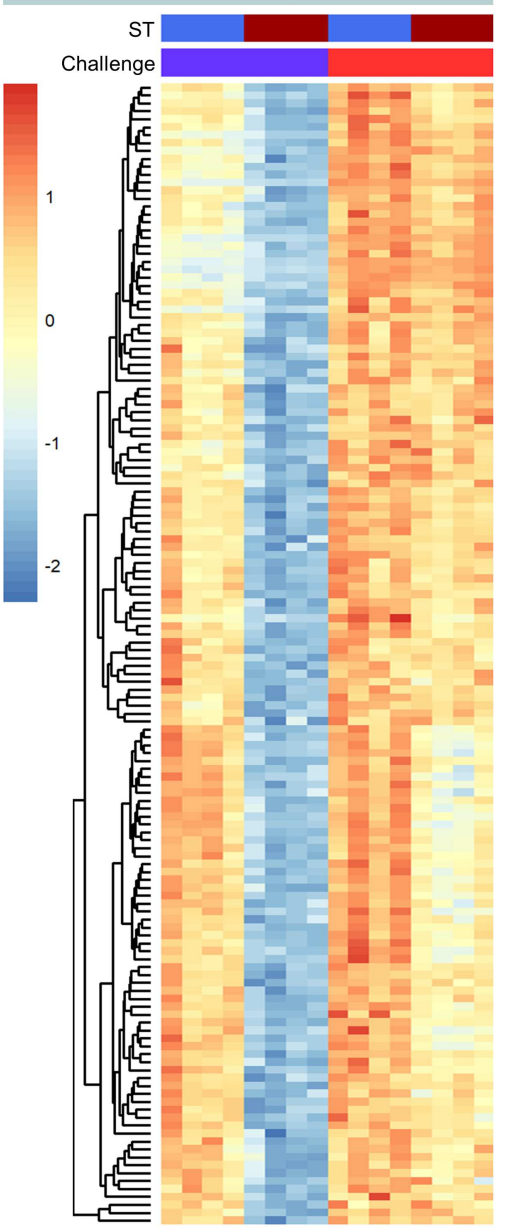

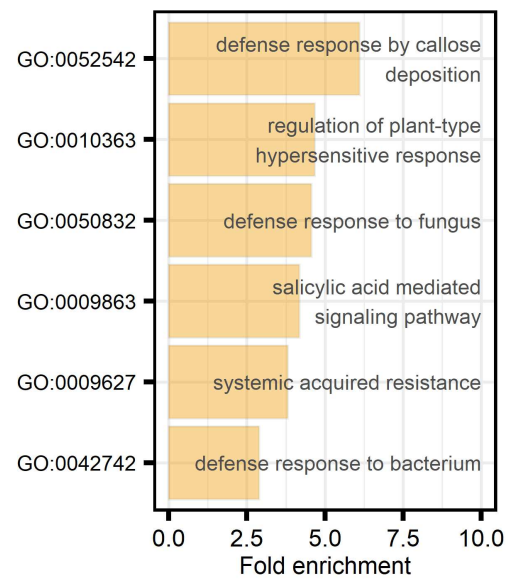

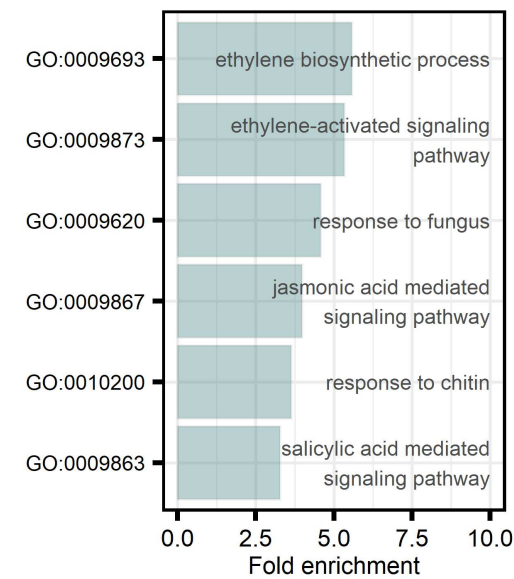


1018

1019

1020

1021

1022

1023

1024

1025

1026

1027

1028

1029

1030

1031

1032

1033

\section{Fig. 2 | Transcriptome of long-term JA-IR against herbivory and JA-IS against}

pathogens. a,b, Principal component analysis (PCA) and hierarchical cluster analysis (HCA) of global gene expression patterns, respectively. Samples for mRNA-seq analysis were collected from 5-week-old plants at 4 hrs after challenge with water (W) or $0.1 \mathrm{mM} \mathrm{JA}$. Plants had been pre-treated with water or $1 \mathrm{mM} \mathrm{JA}$ at the seedling stage (2-weeks-old). Letters before and after the underscore in the heatmap labels indicate seedling treatment and challenge treatment, respectively. c, Transcript profiles of 203, 796 and 144 genes correlating with long-term JA-IR to SI, JA-IS to Pst and JA-IS to PC, respectively. Genes are from gene clusters selected based on expression profile and enrichment of biologically relevant GO terms. For details, see text and Extended Data Figs. 2 and 3. Blue and red columns above the heatmaps indicate water and JA treatments, respectively, of seedlings (ST) and 5-week-old plants (Challenge). Heatmap-projected values represent per gene zscores of transformed read counts from 4 biological replicates for each treatment combination. d, Selection of defence-related Gene Ontology (GO) terms enriched within the sets of IR- or IS-related genes ( $p . a d j<0.05)$. For complete lists of all enriched GO terms, see Supplementary Data 5, 9 and 13. 
a

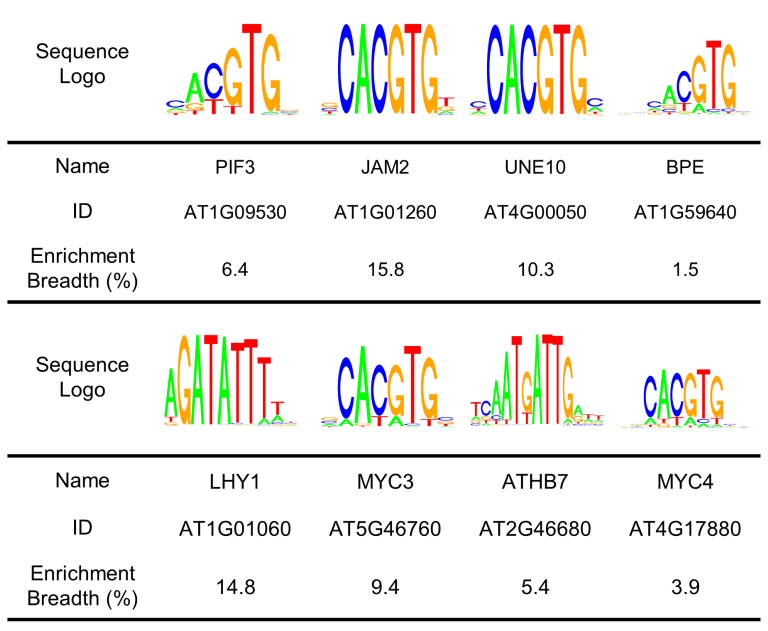

b

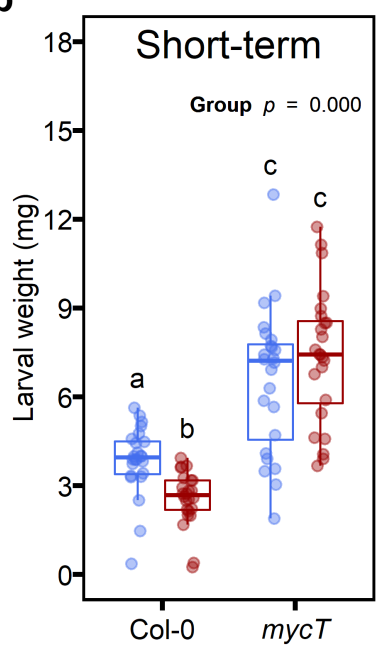

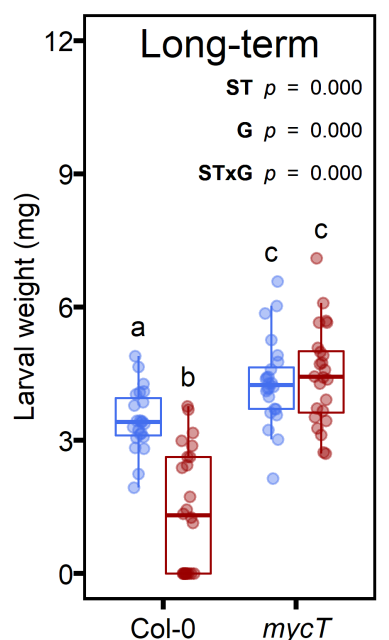

Fig. 3 | MYC2/3/4 transcription factors control short- and long-term JA-IR against

herbivory. a, Statistical enrichment of transcription factor (TF) DNA binding motifs $(p<0.01)$

in the $1 \mathrm{~kb}$ upstream promoter sequences of the 203 IR-related genes (Fig. 2c). Displayed

are the 8 motifs with the strongest statistical enrichment. Enrichment breadth indicates the \% of promoters for which the motif fell within the top $5 \%$ of most strongly enriched motifs. Name and ID indicate predicted TF binding to the DNA motif. For the complete list of all statistically enriched DNA motifs, see Supplementary Data 14. b, Short- and long-term effects of water (blue) and $1 \mathrm{mM} \mathrm{JA} \mathrm{(red)} \mathrm{pre-treatment} \mathrm{on} \mathrm{resistance} \mathrm{of} \mathrm{5-week-old} \mathrm{WT}$ (Col-0) and myc2 myc3 myc4 (mycT) plants against herbivory by Spodoptera littoralis (Sl; $n=23-24)$. Pre-treatment and genotype combinations which do not share the same letter are significantly different (Kruskal-Wallis test followed by pairwise Wilcoxon rank sum tests for short-term or ANOVA followed by Tukey post-hoc test for long-term; p.adj < 0.05). For more details, see legend to Fig. 1c,d. 
a

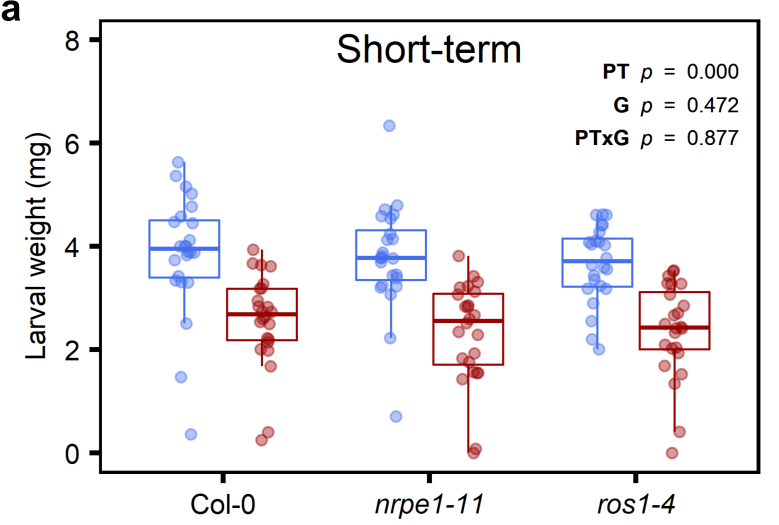

b

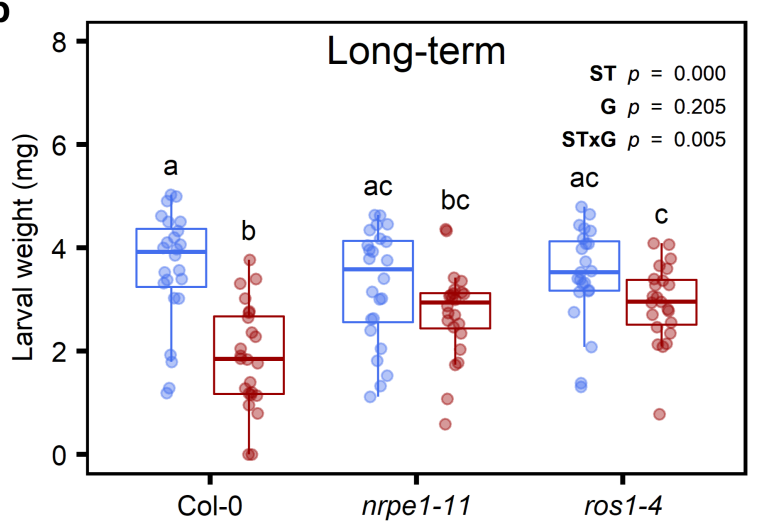

C

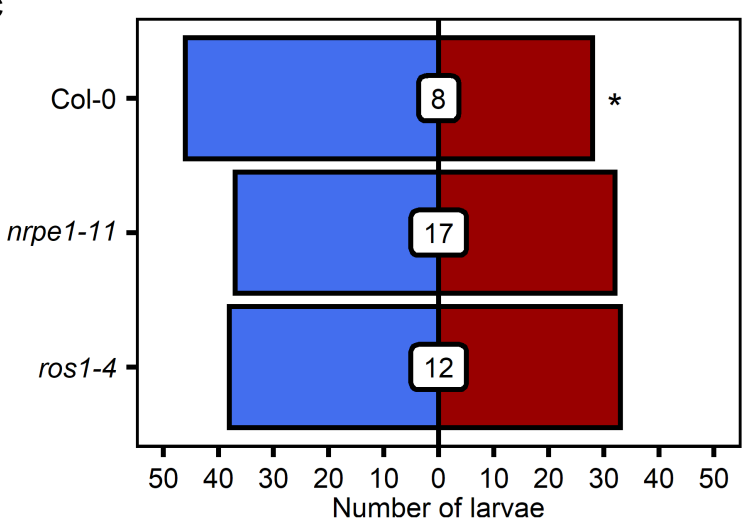

d

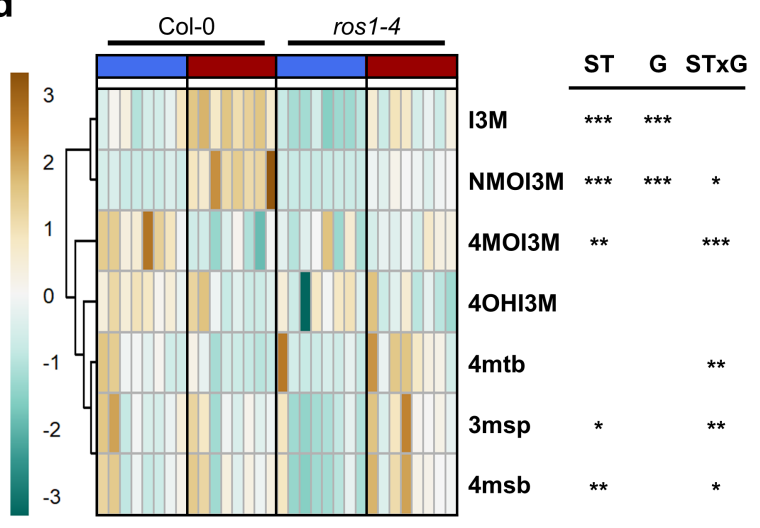

e

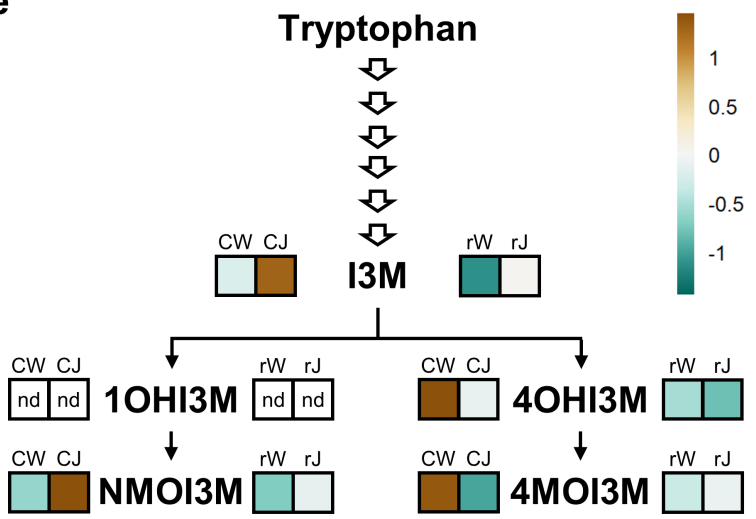

Methionine

$n$
$\frac{n}{n}$
$\frac{n}{n}$
$\frac{n}{n}$
$\frac{n}{n}$

Glucosinolate core structure

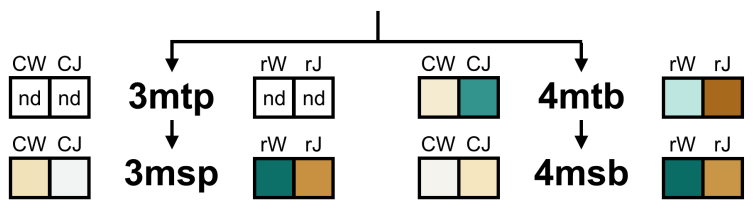

Fig. 4 | Long-term JA-IR against herbivory and associated shifts in glucosinolate

profiles require intact DNA methylation homeostasis. a,b, Short- (a) and long-term (b)

effects of water (blue) and $1 \mathrm{mM} \mathrm{JA} \mathrm{(red)} \mathrm{pre-treatment} \mathrm{on} \mathrm{resistance} \mathrm{of} \mathrm{5-week-old} \mathrm{WT}$

(Col-0) and RdDM (nrpe1-11) and ROS1 (ros1-4) mutant plants against herbivory by

Spodoptera littoralis ( $S I, n=23-24)$. If the pre-treatment (PT) or seedling treatment $(\mathrm{ST}) \mathrm{x}$

1052 Genotype $(\mathrm{G})$ interaction term was significant (Two-way ANOVA, $p<0.05$ ), a Tukey post-

1053 doc test was conducted with different letters indicating significant differences between

1054 means $(p<0.05)$. For more details, see legend to Fig. 1. c, Effects of long-term JA-IR on 
1055 attractiveness to S/ larvae in dual-choice tests. Shown are the number of larvae preferring 51056 week-old plants that had been pre-treated with either water (blue) or $1 \mathrm{mM} \mathrm{JA}$ (red) at the 1057 seedling stage (2-week-old). White boxes indicate larvae failing to make a choice. Asterisks 1058 indicate statistically uneven distributions of larval numbers between treatments (Goodness1059 of-fit test, $\left.{ }^{*} p<0.05\right)$. d, Long-term effect of water and $1 \mathrm{mM}$ JA on all glucosinolates 1060 detected in the leaf tissue of 5-week-old WT and ros 1-4 plants. Heatmap-projected values 1061 represent per metabolite z-scores of concentrations ( $\mu \mathrm{g} / \mathrm{g}$ dry mass) from 8 biological 1062 replicates for each genotype-treatment combination. See Extended Data Fig. 5 for raw data. 1063 Asterisks indicate significant effects of ST, G or ST x G (Two-way ANOVA, * $=p<0.05,{ }^{* *}=$ $1064 p<0.01{ }^{* * *}=p<0.001$ ). e, Biosynthesis pathways of indole (top) and aliphatic (bottom) 1065 glucosinolates. Heatmap-project values represent z-scores of mean concentrations ( $\mu \mathrm{g} / \mathrm{g}$ dry 1066 mass). CW: Col-0 + water ST, CJ: Col-0 + JA ST, rW: ros1-4 + water ST, rJ: ros1-4 + JA ST,

1067 nd: not detected, I3M: glucobrassicin, 1OHI3M: 1-hydroxyglucobrassicin, 4OHI3M: 4-

1068 hydroxyglucobrassicin, 4MOI3M: 4-methoxyglucobrassicin, NMOI3M: neoglucobrassicin, 1069 3mtp: 3-methylthiopropyl glucosinolate, 3msp: glucoiberin, 4mtb: glucoerucin, 4msb:

1070 glucoraphanin. 
a

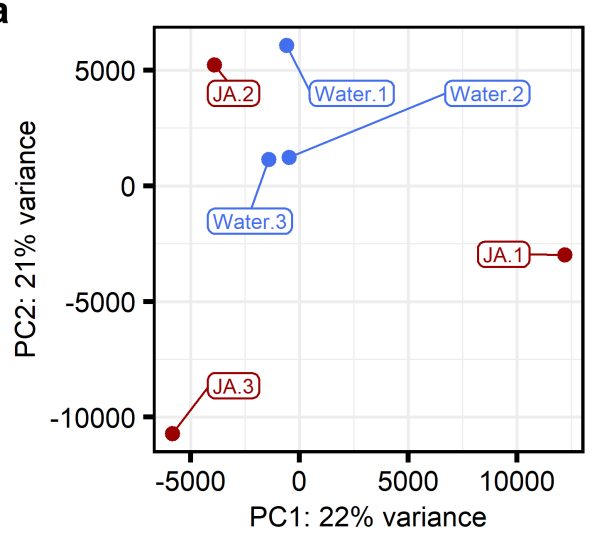

b

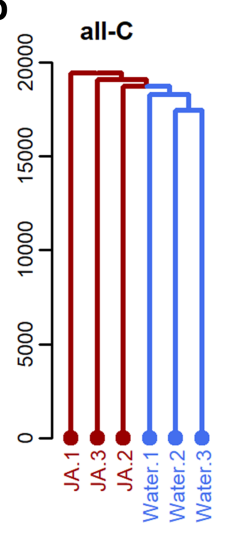

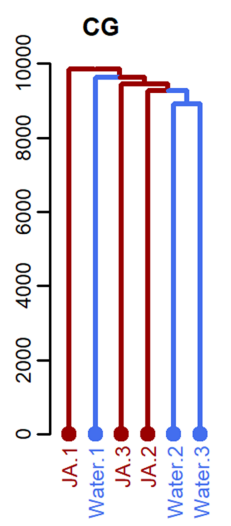
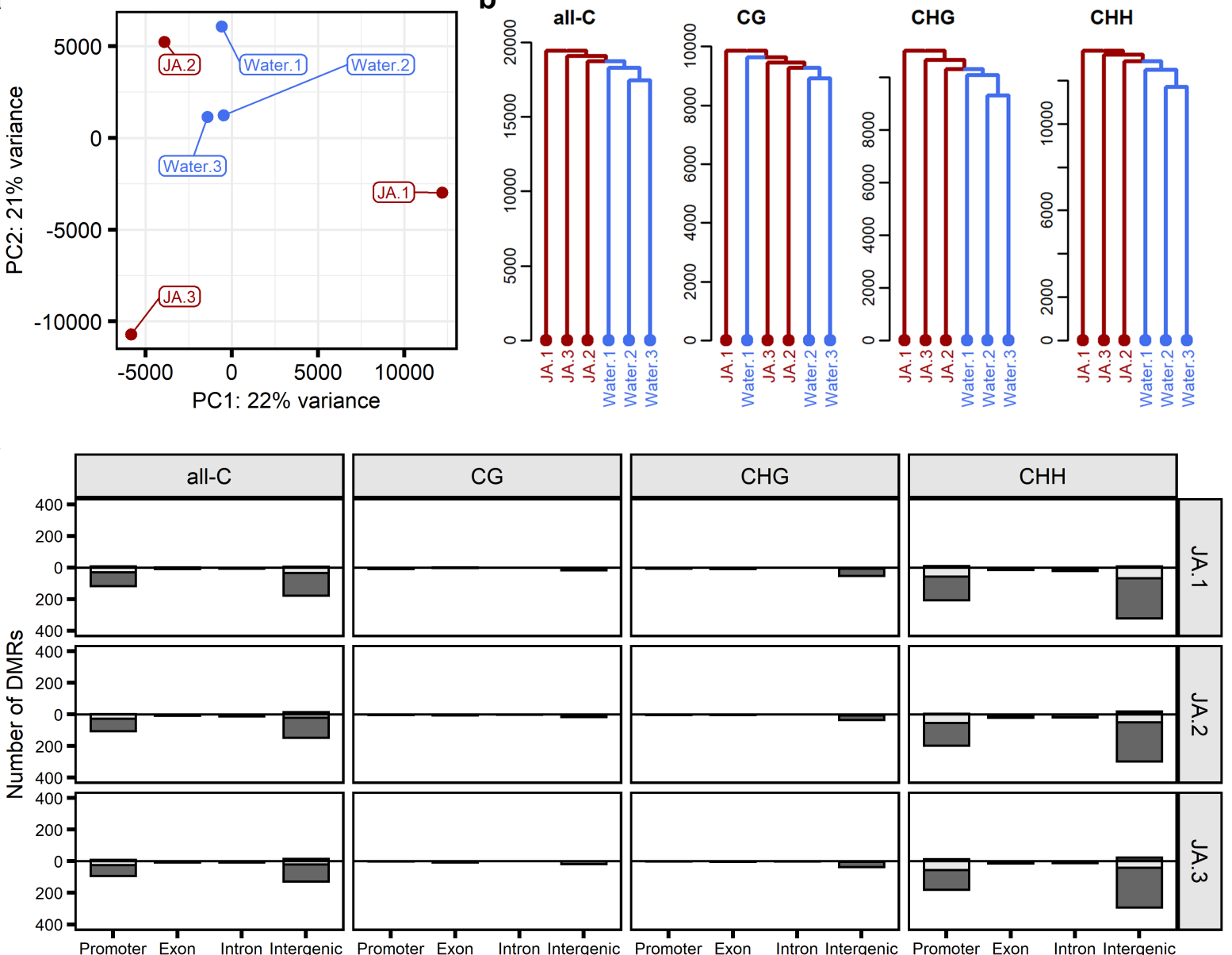

d

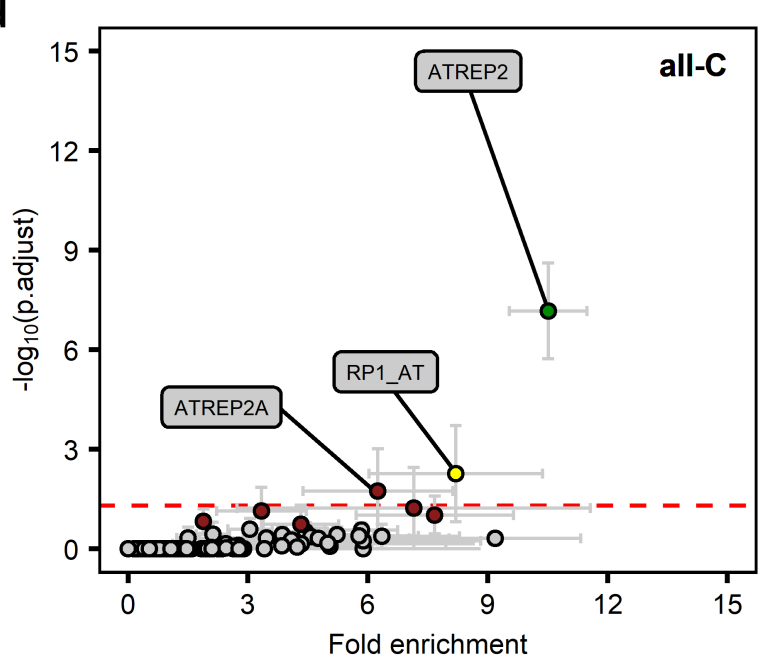

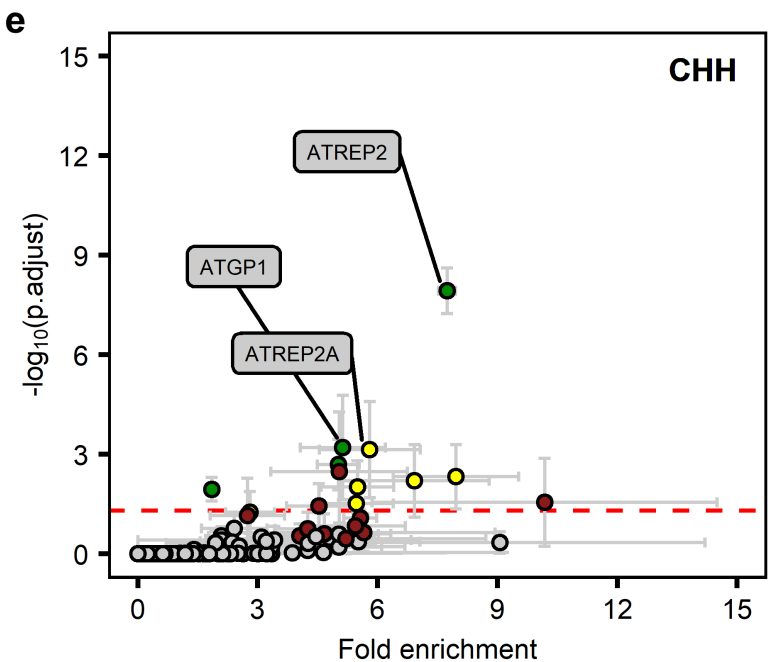

Fig. 5 | The DNA methylome of long-term JA-IR is associated with selective

1072 hypomethylation of ATREP2 transposable elements. Biologically replicated leaf samples

$1073(n=3)$ for whole-genome bisulphite sequencing were collected from 5-week-old plants that

1074 had been pre-treated with water or $1 \mathrm{mM} \mathrm{JA}$ at the seedling stage (2-week-old). a, PCA plot

1075 displaying variation in global cytosine $(\mathrm{C})$ methylation at all-C sequence context between 
1076

1077

1078

1079

1080

1081

1082

1083

1084

1085

1086

1087

1088

1089

1090

samples from water (blue) and JA (red) pre-treated plants. b, HCA plots displaying global variation in $\mathrm{C}$ methylation at all-C, CG, $\mathrm{CHG}$ and $\mathrm{CHH}$ contexts $(\mathrm{H}$ is any nucleotide other than G). c, Numbers of differentially methylated regions (DMRs) between individual samples from JA-treated plants (JA.1, JA.2 and JA.3) and all three samples from water-treated plants (1JA_vs_3W comparisons) at gene promoters, exons, introns and intergenic regions.

Frequencies of hyper- and hypo-methylated DMRs are indicated by the bars above and below the x-axis, respectively. DMRs at transposable elements (TE) are indicated by dark shading. d,e, Enrichment of TE families amongst the TEs overlapped by DMRs at all-C and $\mathrm{CHH}$ contexts, respectively. Graphs plot statistical enrichment of each TE family against its corresponding fold-enrichment, represented by mean $-\log _{10}(p . a d j)$ values $( \pm S E M)$ and mean fold enrichment values ( \pm SEM), respectively. Enrichment is expressed relative to the background of all genome annotated TEs (TAIR v10). Labelled data points indicate TE families with a mean $-\log _{10}\left(\right.$ p.adj) $>-\log _{10}(0.05)(\mathbf{d})$ or $-\log _{10}(0.001)(\mathbf{e})$. Brightly coloured data points indicate TE families that were significantly overrepresented in 1 (red), 2 (yellow) or 3 (green) comparisons, respectively $(p . a d j \leq 0.05)$. The red dashed line is at $-\log _{10}(0.05)$. 
a

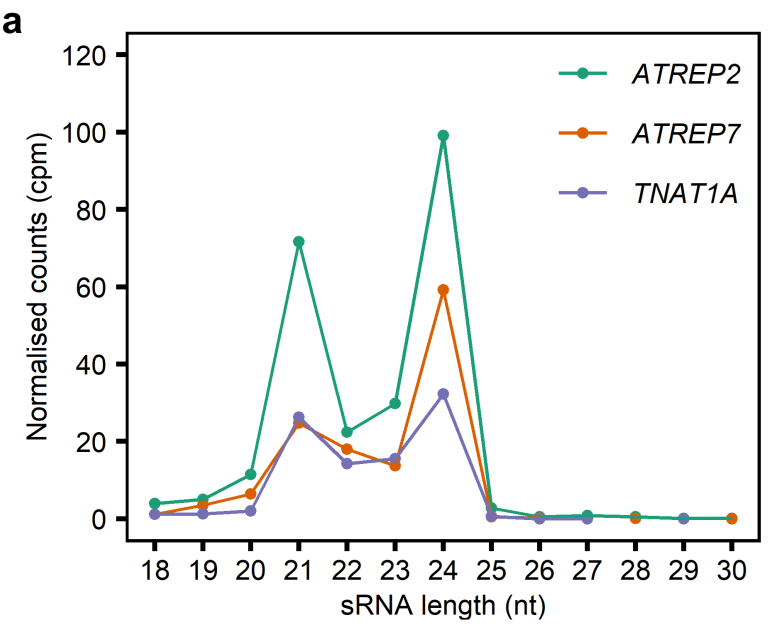

C

Naive b

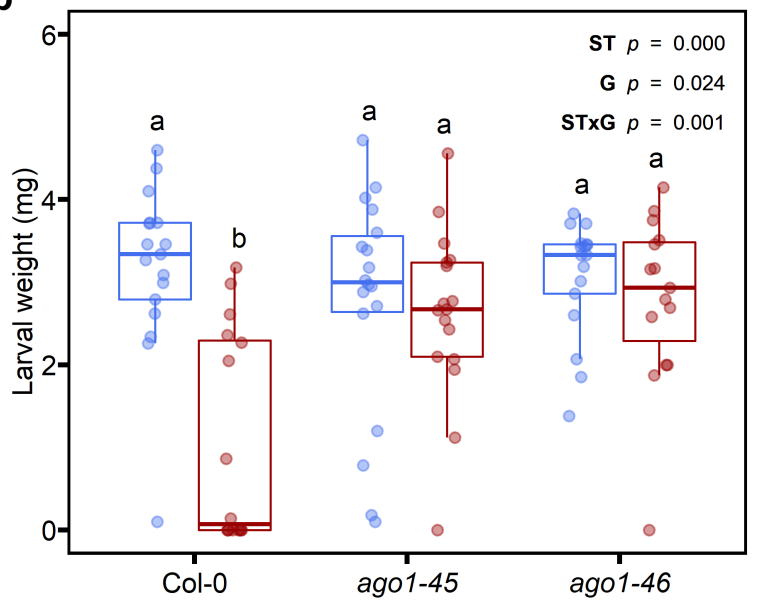

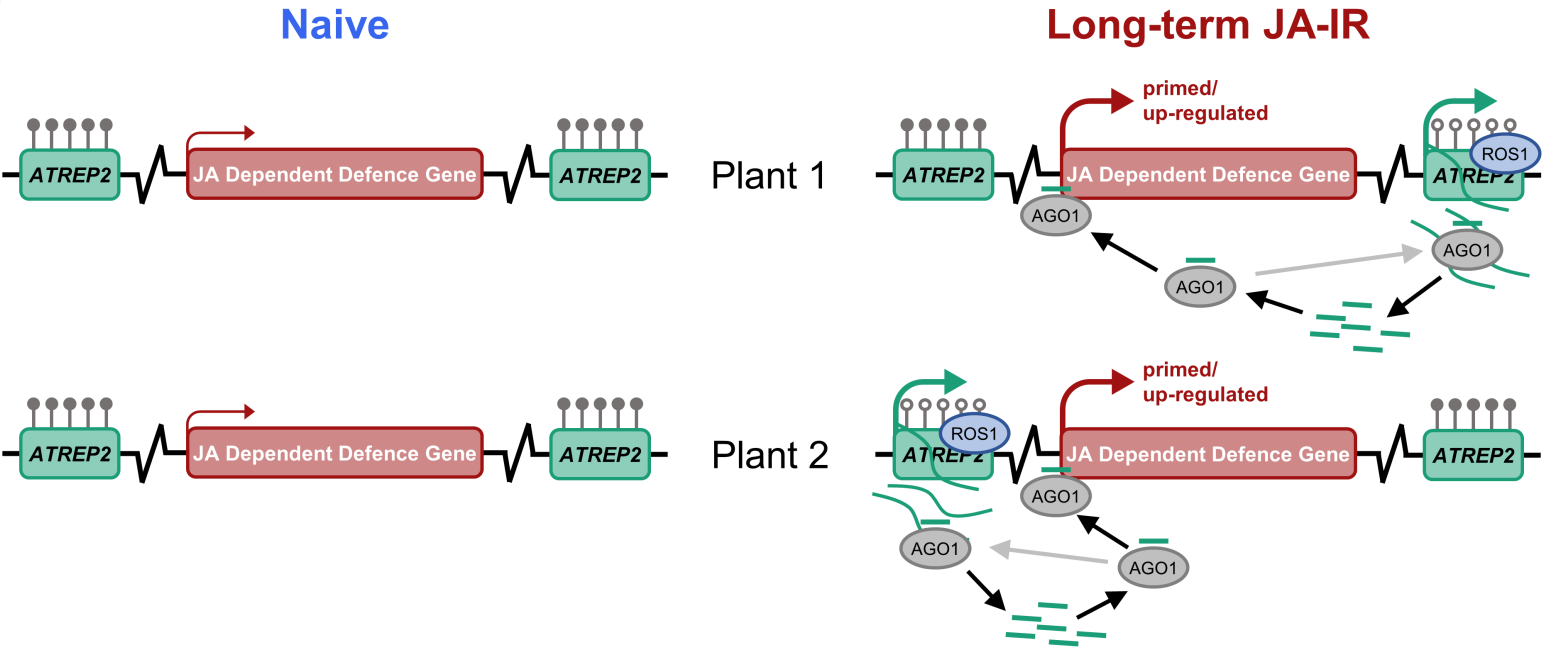

Fig. 6 | AG01 shows increased association with small interfering RNAs (siRNAs) from

ATREP2 TEs and is essential for long-term JA-IR against herbivory. a, Frequency-size distributions of AGO1-associated small RNAs (sRNAs) mapping to TEs from the ATREP2 family compared to sRNAs mapping to similarly sized TE families that were not targeted for hypomethylation by JA seedling treatment (ATREP7 and NTAT1A). Nuclear AGO1 was extracted from 10-day-old seedlings at $1 \mathrm{~h}$ after treatment with $50 \mu \mathrm{M} \mathrm{MeJA}{ }^{42}$. To enrich the dataset with siRNAs, reads from other known classes of RNAs were excluded from the analysis. All three TE families belong to class 2 and have similar numbers in the Arabidopsis genome (162-164). Counts of sRNAs ranging from 18-30 nucleotides (nt) are displayed as counts per million (cpm) reads. $\mathbf{b}$, Long-term effects of water (blue) and $1 \mathrm{mM} \mathrm{JA}$ (red) seedling treatment on resistance of 5-week-old WT (Col-0) and ago1 plants against 
1103 the seedling treatment (ST) x Genotype (G) interaction term was significant (Two-way

1104 ANOVA, $p<0.05)$, a Tukey post-doc test was conducted, with different letters indicating

1105 significant differences between means $(p<0.05)$. c, Model of trans-regulation of long-term

1106 JA-IR by hypomethylated ATREP2 TEs and AGO1. Following JA seedling treatment,

1107 members of the ATREP2 TE family remain stochastically hypomethylated by ROS1.

1108 Hypomethylated ATREP2 TEs generate transcripts (long curved green lines) that are

1109 cleaved by post-transcriptional gene silencing (PTGS) machinery to siRNAs (short green

1110 lines) that associate with AG01. Some siRNA-associated AGO1 proteins associate with the

1111 chromatin of distant defence genes, where they recruit Pol-II to upregulate and/or prime

1112 expression. 


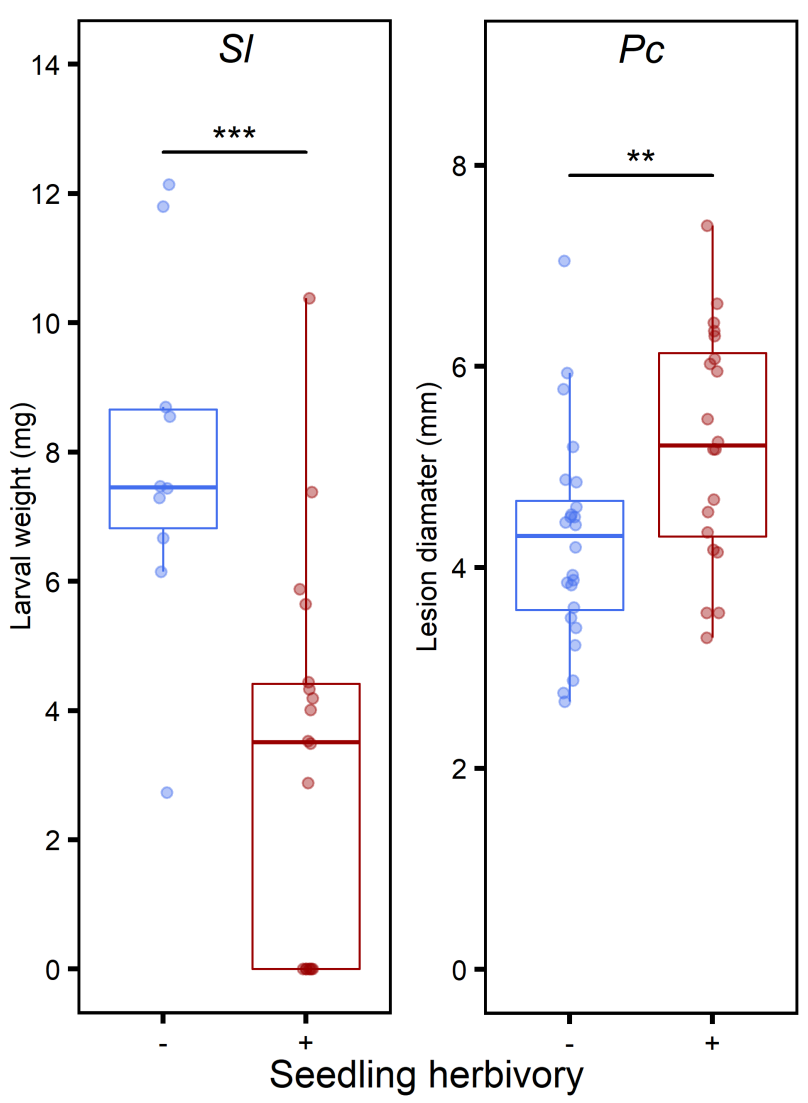

1113 Extended Data Fig. 1 | Herbivore damage at the seedling stage results in long-term IR

1114 against herbivory and long-term IS against necrotrophic pathogen infection. Effect of

1115 feeding damage by Spodoptera littoralis $(S /)$ larvae at the 2-week-old seedling stage on the

1116 resistance of 5-week-old Arabidopsis (Col-0) against herbivory by S/ larvae (left panel) and

1117 disease by necrotrophic Plectosphaerella cucumerina (PC; right panel). Data points

1118 represent weights of individual $S /$ larvae after feeding on individual plants $(n=10-18)$ or

1119 average per plant lesion diameters by $P c(n=18-21)$. Asterisks indicate statistically significant

1120 differences between seedling treatments (Mann-Whitney test for S/ or two sample t-test for

$\left.1121 P C ;{ }^{* *} p<0.01,{ }^{* * *} p<0.001\right)$. For more details about experimental design, see legend for

1122 Fig. 1. 


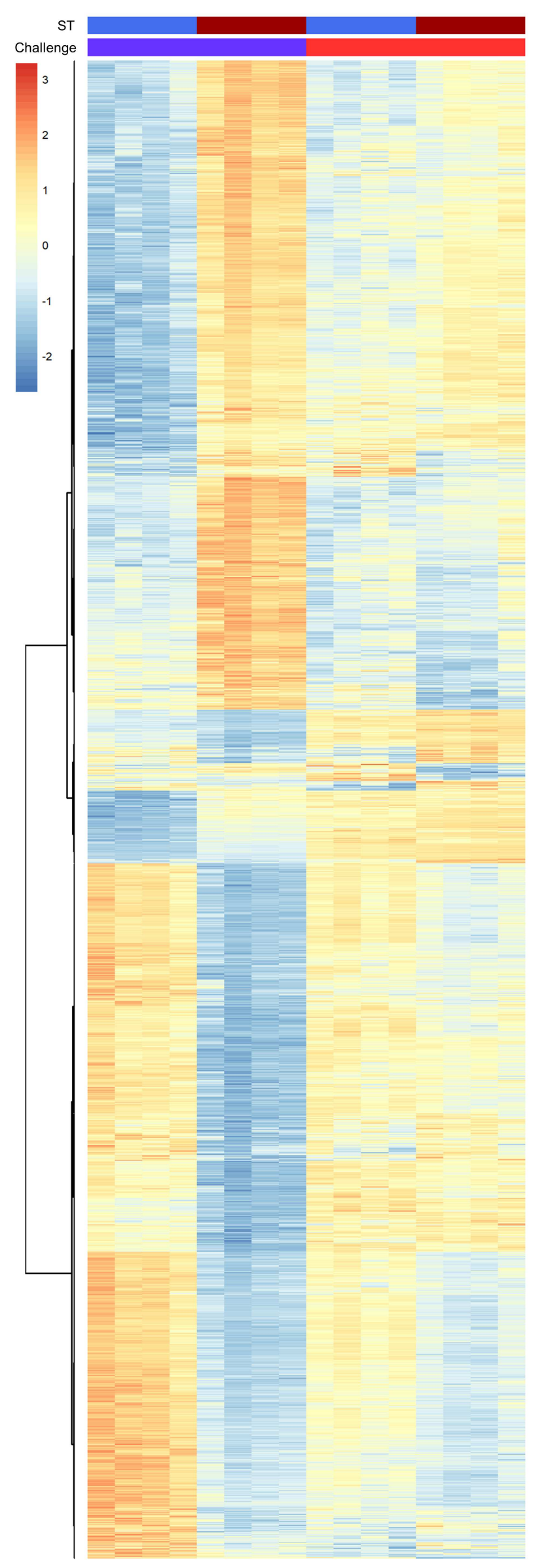

Extended Data Fig. 2 | Selection of genes showing altered JA responsiveness to JA challenge in 5week-old plants as a consequence of JA seedling treatment. Expression profiles of 2,409 genes with a statistically significant interaction between seedling treatment (ST) and challenge treatment (p.adj < 0.01). Replicate samples $(n=4)$ for mRNA-seq analysis were collected from 5week-old plants at $4 \mathrm{hrs}$ after challenge with water $(\mathrm{W})$ or $0.1 \mathrm{mM}$ JA. Plants had been pre-treated with water or $1 \mathrm{mM} \mathrm{JA}$ at the seedling stage (2-week-old). Blue and red columns above the heatmap indicate water and JA treatments, respectively. Heatmap-projected values represent per gene z-scores of transformed read counts from all biological replicates. 
a

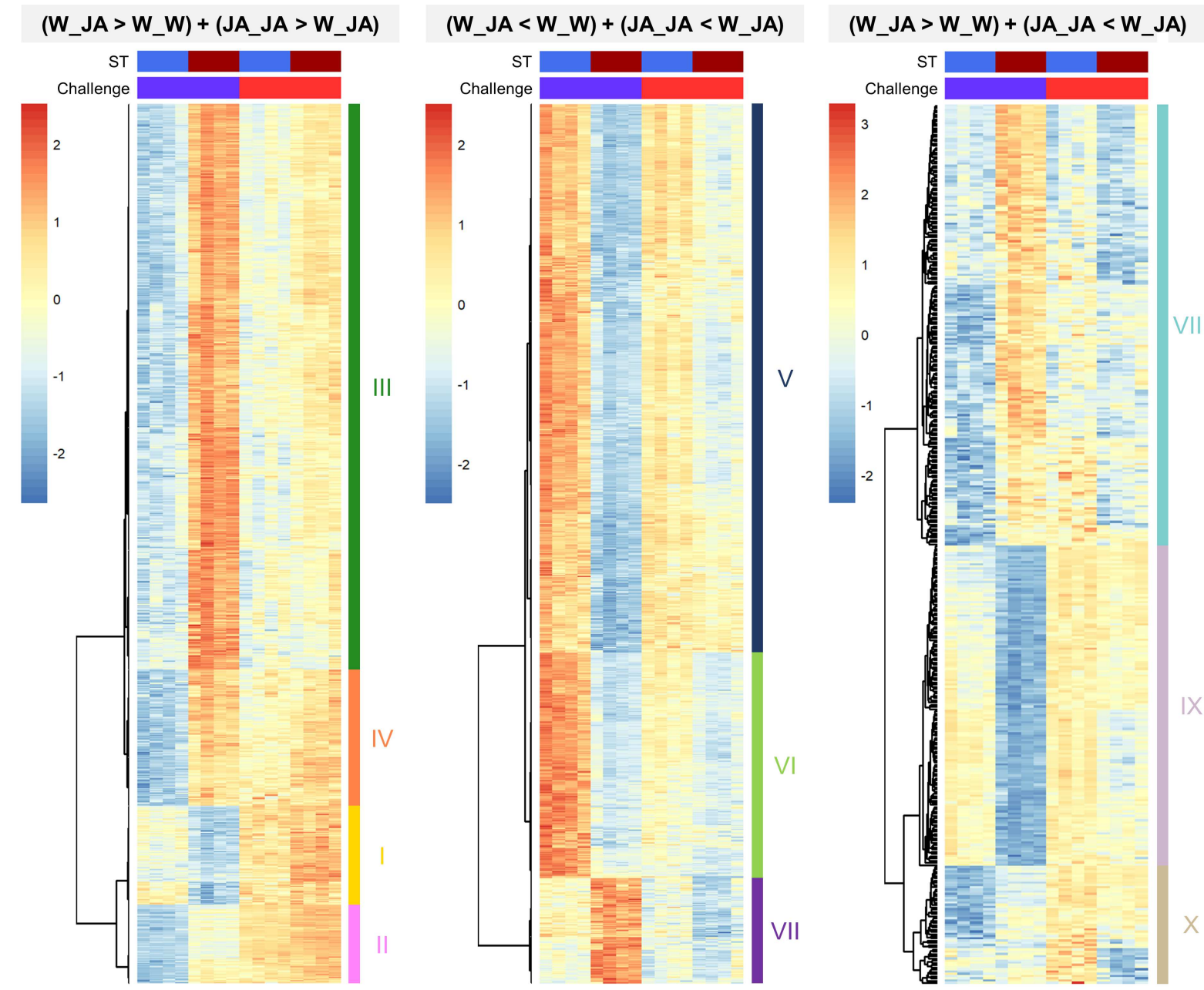

b

\begin{tabular}{|c|c|c|c|}
\hline Cluster & GO term & GO name & $\begin{array}{c}\text { Fold } \\
\text { enrichment }\end{array}$ \\
\hline ॥ & GO:0009694 & $\begin{array}{l}\text { jasmonic acid } \\
\text { metabolic process }\end{array}$ & 9.60 \\
\hline II & GO:0019760 & $\begin{array}{l}\text { glucosinolate } \\
\text { metabolic process }\end{array}$ & 8.97 \\
\hline ॥ & GO:0009753 & $\begin{array}{l}\text { response to } \\
\text { jasmonic acid }\end{array}$ & 7.68 \\
\hline II & GO:0009611 & $\begin{array}{l}\text { response to } \\
\text { wounding }\end{array}$ & 5.50 \\
\hline IV & GO:0019761 & $\begin{array}{l}\text { glucosinolate } \\
\text { biosynthetic } \\
\text { process }\end{array}$ & 7.99 \\
\hline
\end{tabular}

\begin{tabular}{|c|c|c|c|}
\hline Cluster & GO term & GO name & $\begin{array}{c}\text { Fold } \\
\text { enrichment }\end{array}$ \\
\hline $\mathbf{V}$ & GO:0052542 & $\begin{array}{c}\text { defense response } \\
\text { by callose } \\
\text { deposition }\end{array}$ & 4.89 \\
\hline $\mathbf{V}$ & GO:0009863 & $\begin{array}{c}\text { salicylic acid } \\
\text { mediated signaling } \\
\text { pathway }\end{array}$ & 4.27 \\
\hline V & GO:0009627 & $\begin{array}{c}\text { systemic acquired } \\
\text { resistance }\end{array}$ & 3.96 \\
\hline VI & $G 0: 0050832$ & $\begin{array}{c}\text { defense response } \\
\text { to fungus }\end{array}$ & 5.11 \\
\hline VI & GO:0009626 & $\begin{array}{c}\text { plant-type } \\
\text { hypersensitive } \\
\text { response }\end{array}$ & 4.98 \\
\hline VI & GO:0042742 & $\begin{array}{c}\text { defense response } \\
\text { to bacterium }\end{array}$ & 3.04 \\
\hline
\end{tabular}

\begin{tabular}{|c|c|c|c|}
\hline Cluster & GO term & GO name & $\begin{array}{c}\text { Fold } \\
\text { enrichment }\end{array}$ \\
\hline IX & GO:0009693 & $\begin{array}{c}\text { ethylene } \\
\text { biosynthetic } \\
\text { process }\end{array}$ & 5.58 \\
\hline IX & GO:0009873 & $\begin{array}{c}\text { ethylene-activated } \\
\text { signaling pathway }\end{array}$ & 5.33 \\
\hline IX & GO:0009620 & $\begin{array}{c}\text { response to } \\
\text { fungus }\end{array}$ & 4.58 \\
\hline IX & GO:0009867 & $\begin{array}{c}\text { jasmonic acid } \\
\text { mediated signaling } \\
\text { pathway }\end{array}$ & 3.98 \\
\hline IX & GO:0010200 & $\begin{array}{c}\text { response to chitin } \\
\text { restic| }\end{array}$ & 3.63 \\
\hline IX & GO:0009863 & $\begin{array}{c}\text { salicylic acid } \\
\text { mediated signaling } \\
\text { pathway }\end{array}$ & 3.28 \\
\hline
\end{tabular}

1142 Extended Data Fig. 3 | Selection of genes with expression profiles and predicted

1143 functions that correlate with long-term JA-IR against herbivory (left) and long-term

1144 JA-IS against biotrophic (middle) and necrotrophic (right) pathogens. a, Gene

expression profiles were selected from the 2,409 genes with a statistically significant

1146 interaction between seedling and challenge treatment $(p . a d j<0.01)$, using the criteria 
1147 displayed above the heatmaps (letters before and after the underscore indicate seedling

1148 treatment and challenge, respectively), resulting in 832 (left), 904 (middle) and 395 (right)

1149 genes. Replicate samples $(n=4)$ for mRNA-seq analysis were collected from 5-week-old

1150 plants at $4 \mathrm{hrs}$ after challenge with water $(\mathrm{W})$ or $0.1 \mathrm{mM}$ JA. Plants had been pre-treated with

1151 water or $1 \mathrm{mM} \mathrm{JA}$ at the seedling stage (2-week-old). Blue and red columns above the

1152 heatmaps indicate water and JA treatments, respectively, of seedlings (ST) and 5-week-old

1153 plants (Challenge). Heatmap-projected values represent per gene z-scores of transformed

1154 read counts from all biological replicates. Numbered boxes next to heatmaps indicate 10

1155 distinct gene expression clusters. b, Selection of defence-related Gene Ontology (GO) terms

1156 enriched $($ p.adj < 0.05) within the 10 gene clusters shown in $(\mathbf{a})$. For complete lists of

1157 enriched GO terms, see Supplementary Data 3, 7 and 11. 


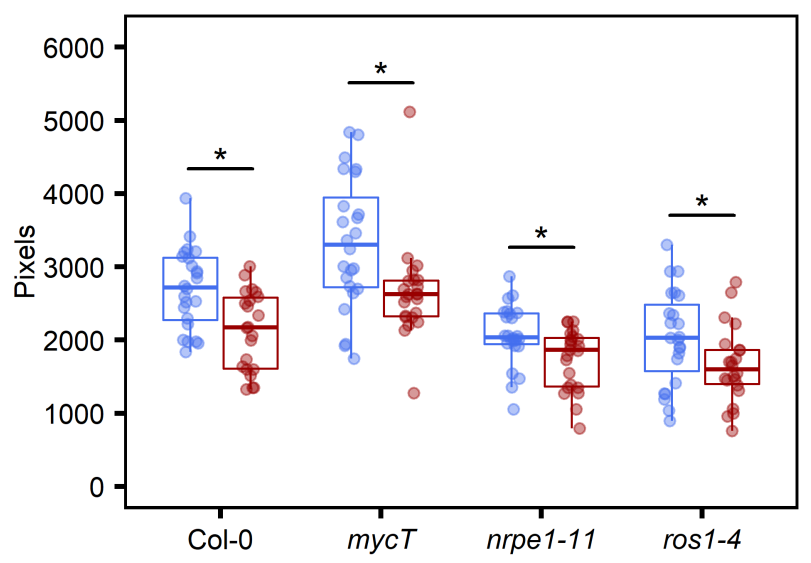

1158 Extended Data Fig. 4 | JA seedling treatment reduces plant growth independently of 1159 MYC2/3/4 TFs and RdDM- and ROS1-dependent regulation of DNA methylation.

1160 Hyperspectral imaging quantified rosette surface areas of 5-week-old plants $(n=22-24)$ pre-

1161 treated with water (blue) or $1 \mathrm{mM} \mathrm{JA}$ (red) at the seedling stage (2-weeks-old). Asterisks

1162 indicate statistically significant within genotype differences between treatments (Wilcoxon

1163 rank sum test, $\left.{ }^{*} p<0.05\right)$. 
a
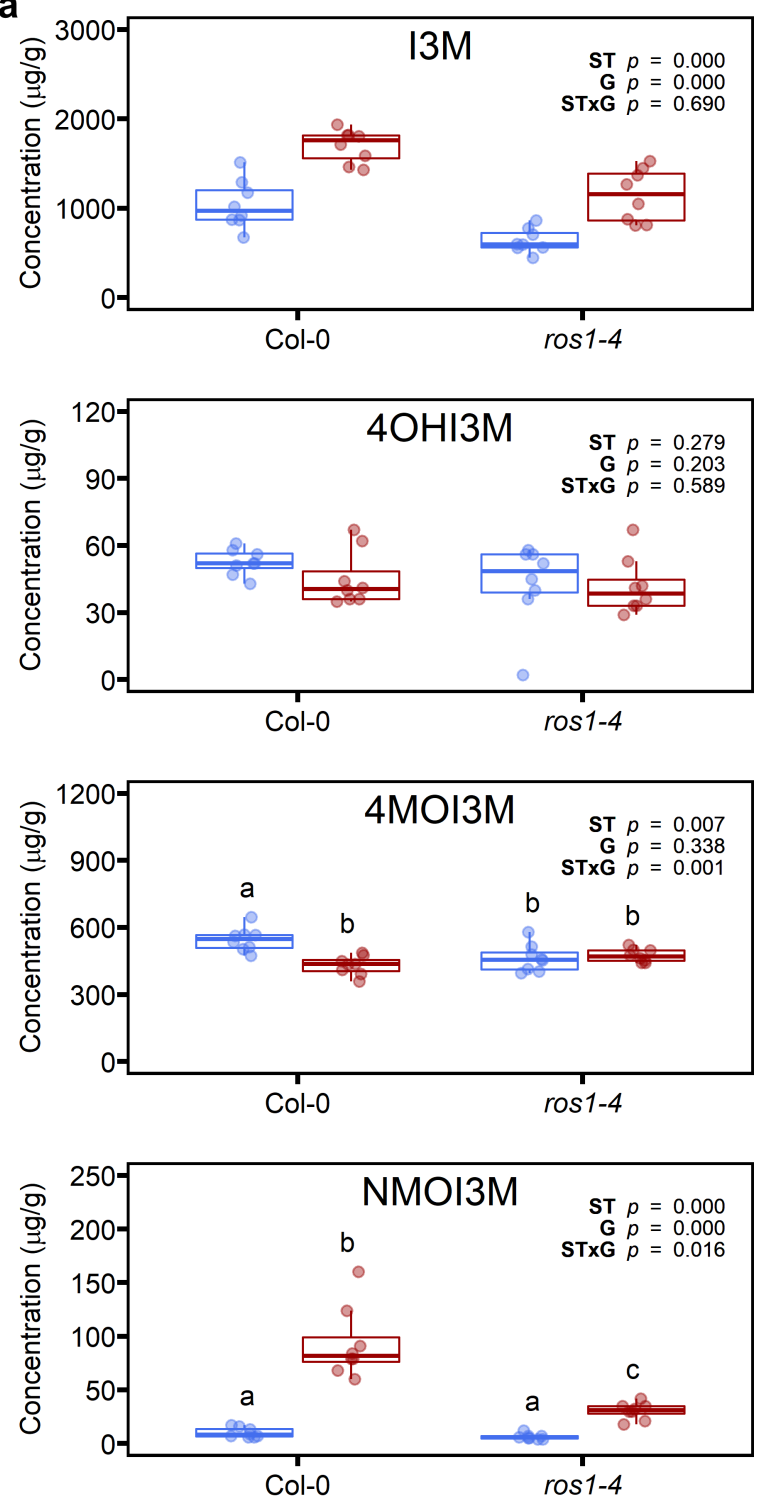

b
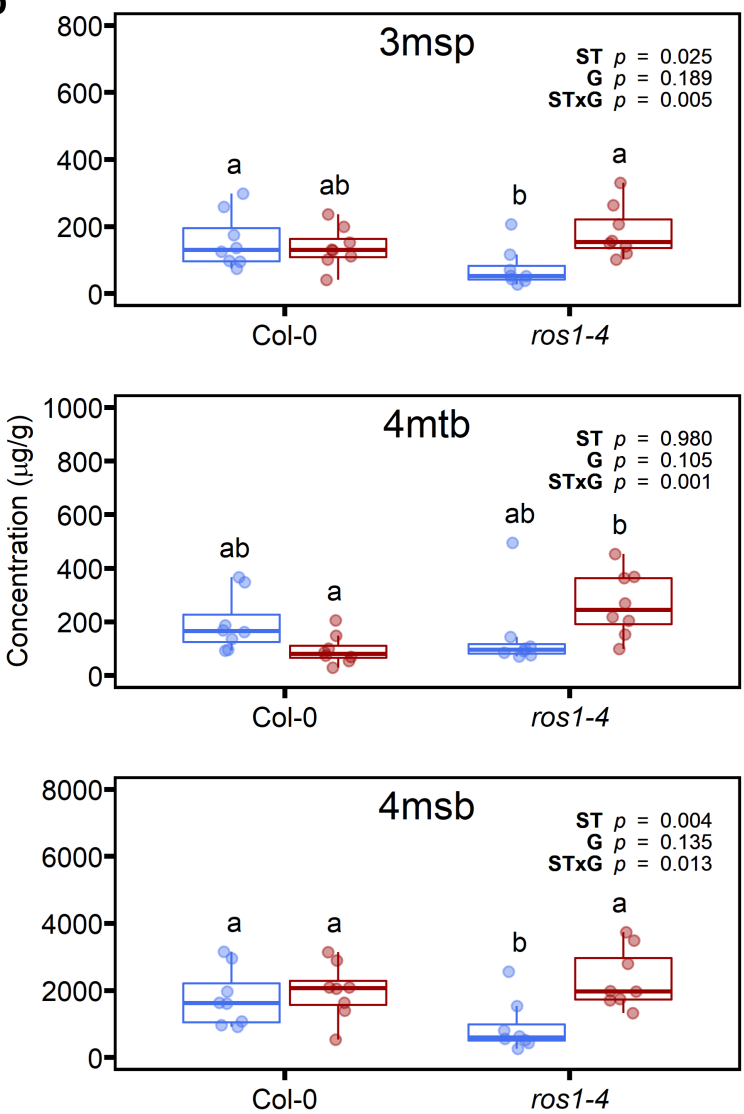

1164

1165

1166

\section{Extended Data Fig. 5 | JA seedling treatment induces long-lasting changes in} glucosinolate content that are dependent on the DNA demethylase ROS1. Long-term effects of water (blue) and $1 \mathrm{mM} \mathrm{JA} \mathrm{(red)} \mathrm{treatments} \mathrm{of} \mathrm{2-week-old} \mathrm{seedlings} \mathrm{on} \mathrm{the}$ concentrations ( $\mu \mathrm{g} / \mathrm{g}$ dry mass) of all glucosinolates detected in the leaf tissue of 5 -week-old WT (Col-0) and ros 1-4 plants ( $n=8)$. a, Indole glucosinolates. b, Aliphatic glucosinolates. If the seedling treatment (ST) x Genotype (G) interaction term was significant (Two-way ANOVA, $p<0.05)$, a Tukey post-doc test was conducted with different letters indicating significant differences between means ( $p<0.05)$. I3M: glucobrassicin, 4OHI3M: 4-

hydroxyglucobrassicin, 4MOI3M: 4-methoxyglucobrassicin, NMOI3M: neoglucobrassicin, 3msp: glucoiberin, 4mtb: glucoerucin, $4 \mathrm{msb}$ : glucoraphanin. 
a

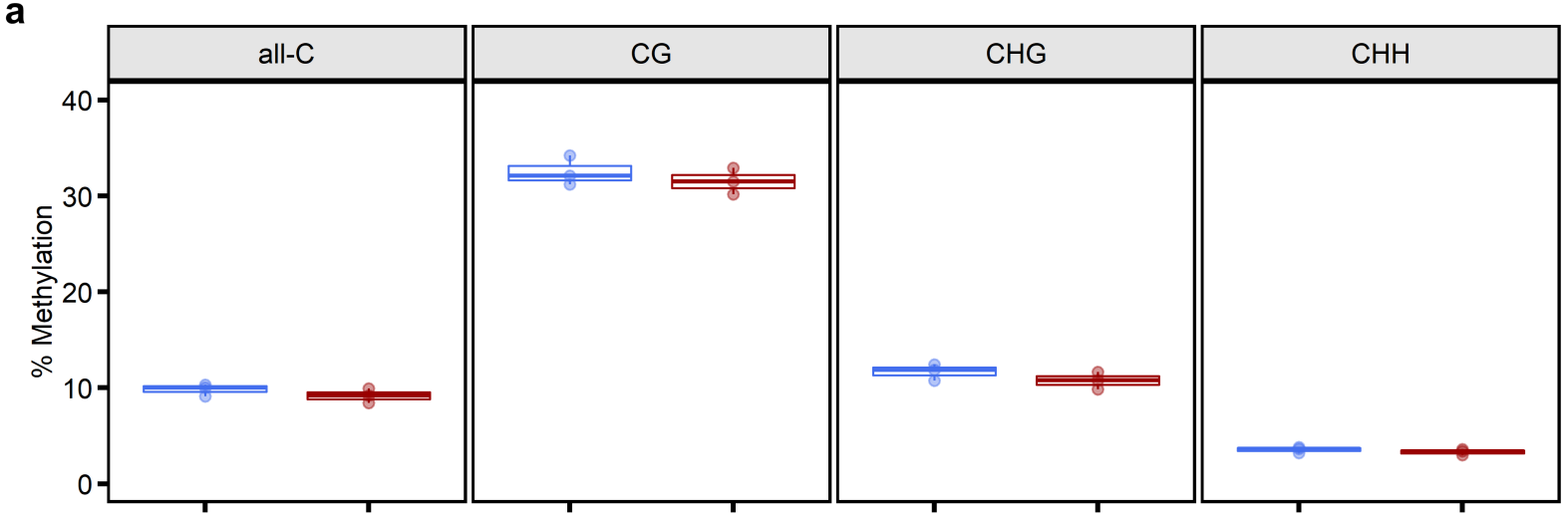

b
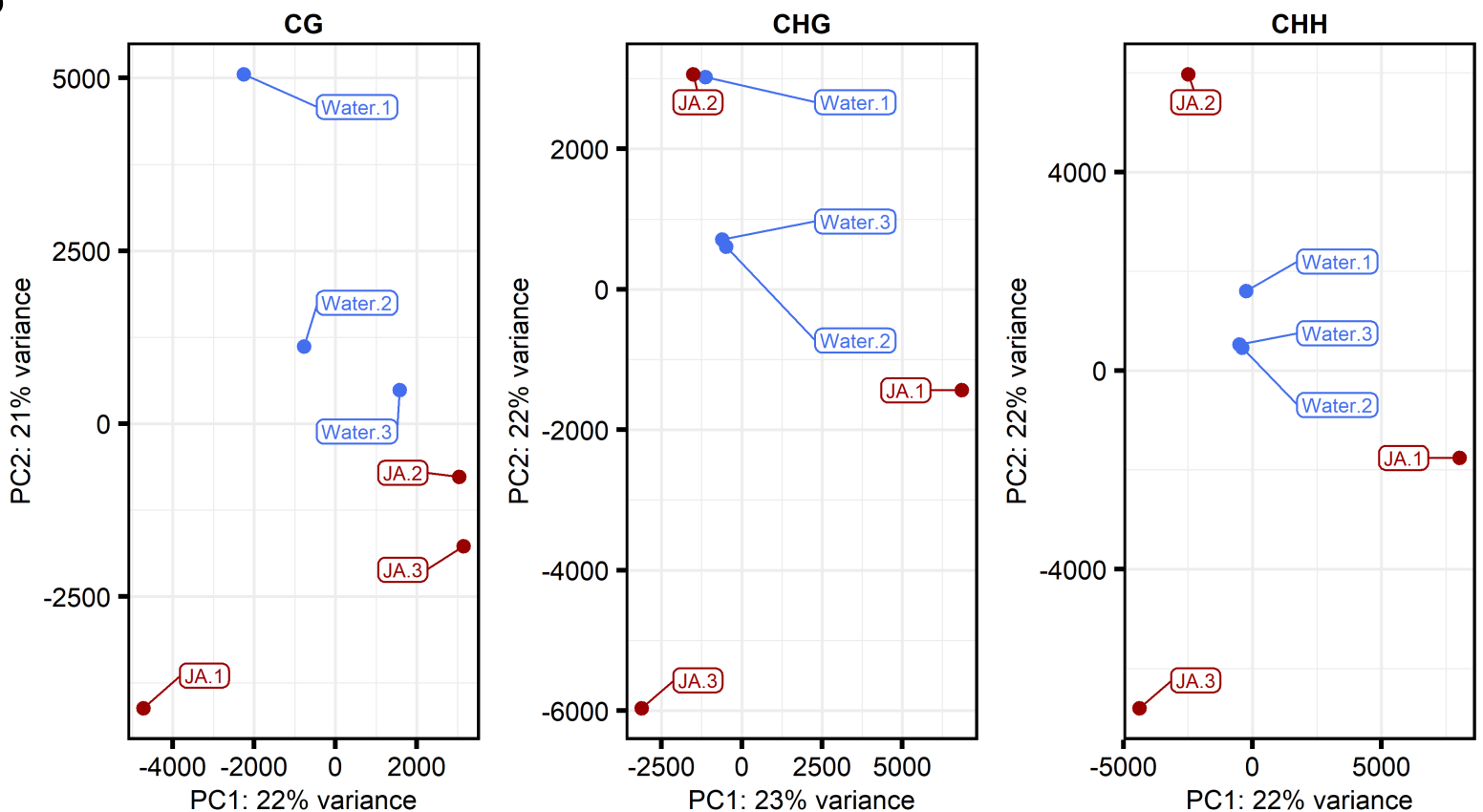

1174 Extended Data Fig. 6 | Long-term impacts of JA seedling treatment on global DNA

methylation levels and patterning. a, Long-lasting effects of JA on global weighted cytosine (C) methylation levels at all-C, CG, CHG and $\mathrm{CHH}$ contexts ( $\mathrm{H}$ indicates any

1177 nucleotide other than G). Data points indicate biologically replicated samples $(n=3)$ from 5-

1178 week-old plants that had been pre-treated with water (blue) or $1 \mathrm{mM} \mathrm{JA}$ (red) at the seedling

1179 stage (2-weeks-old). No statistically significant differences between seedling treatments

1180 were detected (two-sample t-tests, $p>0.05$ ). $\mathbf{b}$, PCA plots of global methylation at CG, CHG 1181 or $\mathrm{CHH}$ contexts. 
a

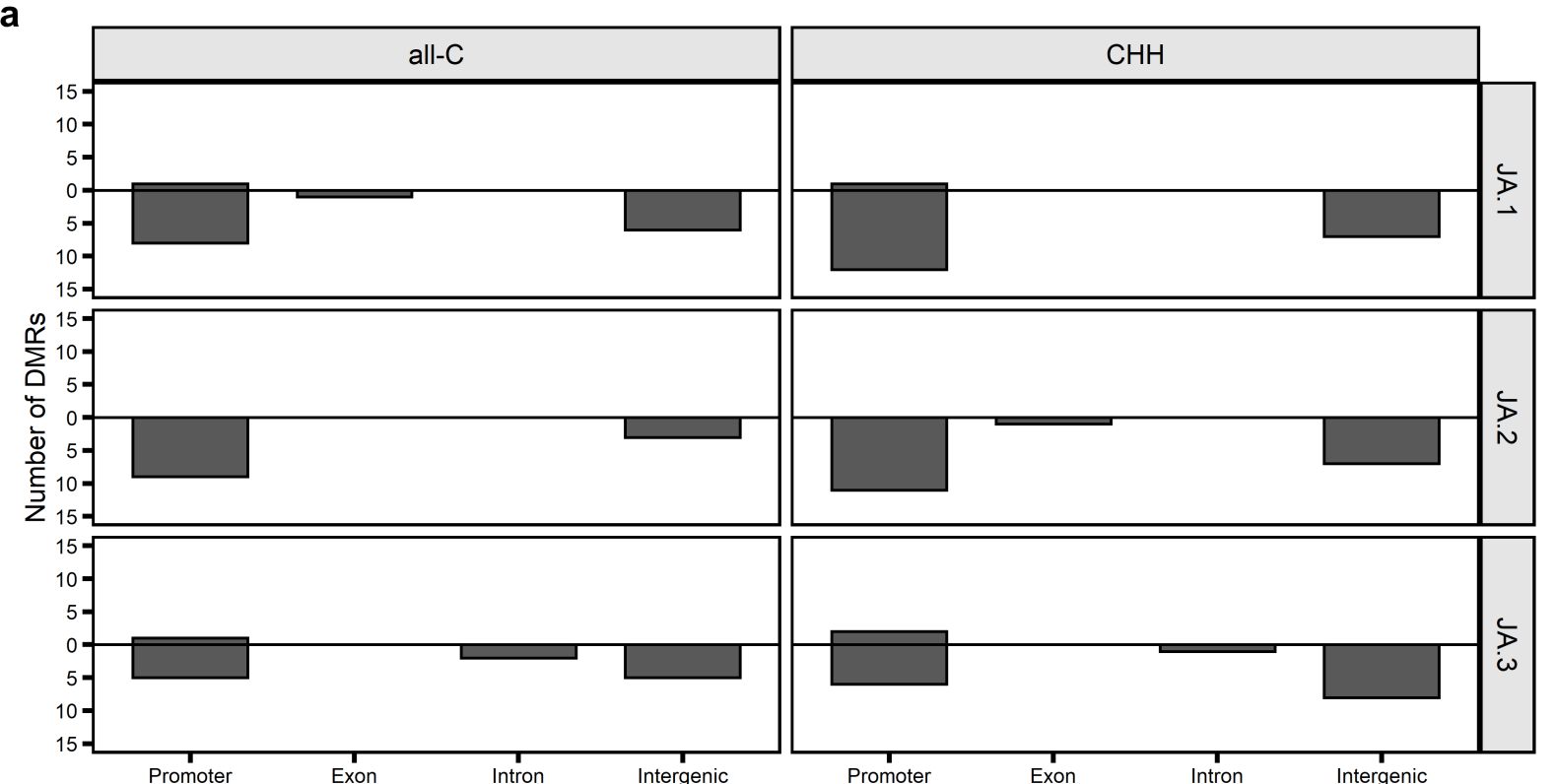

b

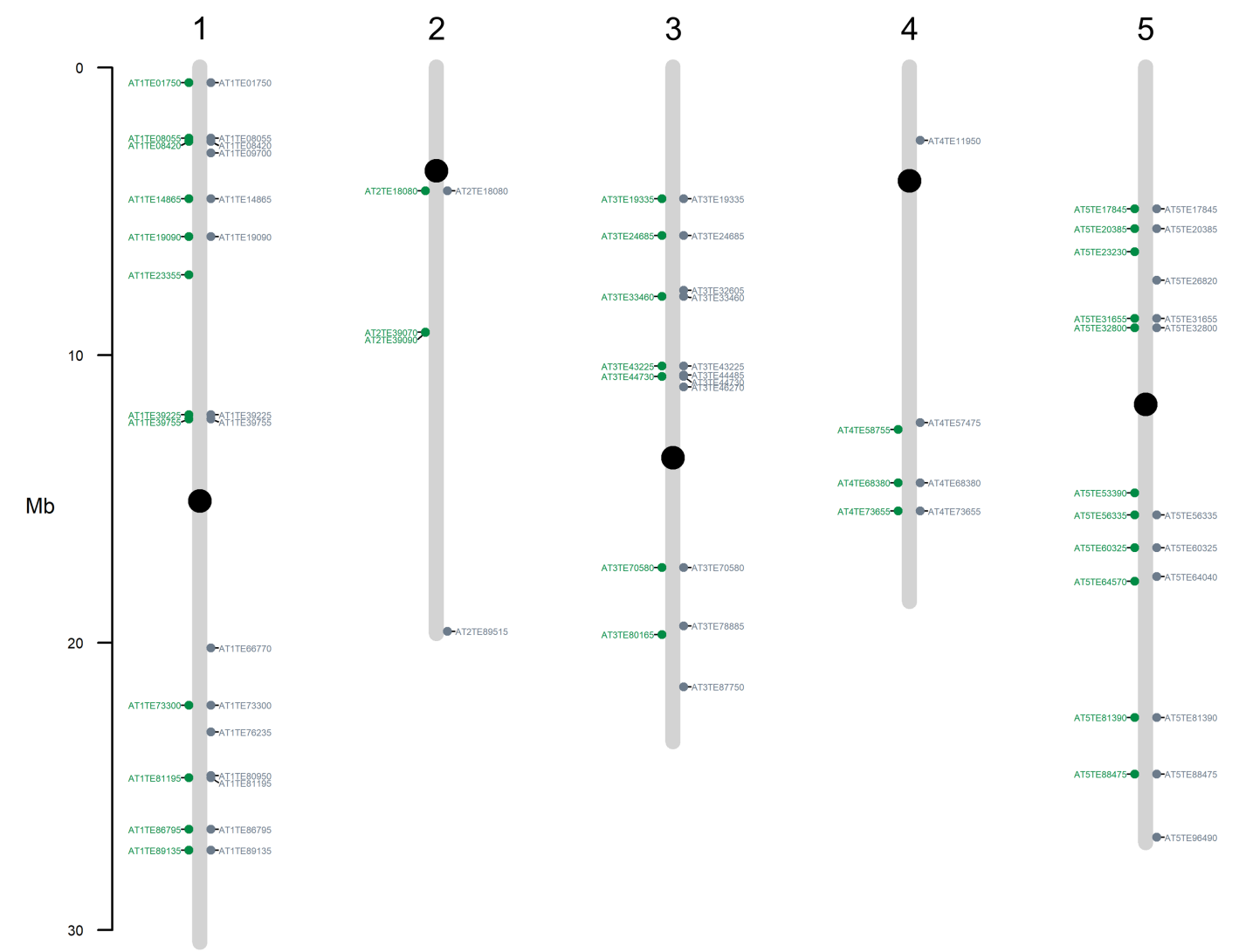

1182 Extended Data Fig. 7 | Differentially methylated regions in ATREP2 transposable

1183 elements are predominantly hypomethylated and spread across the genome. a,

1184 Numbers and genomic contexts of differentially methylated regions (DMRs) overlapping with

1185 ATREP2 transposable elements (TEs). For details about DMR selection, see legend to Fig.

1186 5c. Frequencies of hyper- and hypo-methylated DMRs are indicated by the bars above and 


\section{Wilkinson et al}

1187 below the $\mathbf{x}$-axis, respectively. $\mathbf{b}$, Distribution across the 5 Arabidopsis chromosomes of

1188 DMRs overlapping with ATREP2 TEs. Black dots and grey bars indicate centromeres and

1189 chromosomes, respectively. ATREP2 TEs labelled in green and grey overlapped with DMRs

1190 at all-C or $\mathrm{CHH}$ sequence contexts, respectively. Shown are all ATREP2 TEs which

1191 overlapped with at least one DMR from one 1JA_vs_3W comparison. 
Wilkinson et al

a

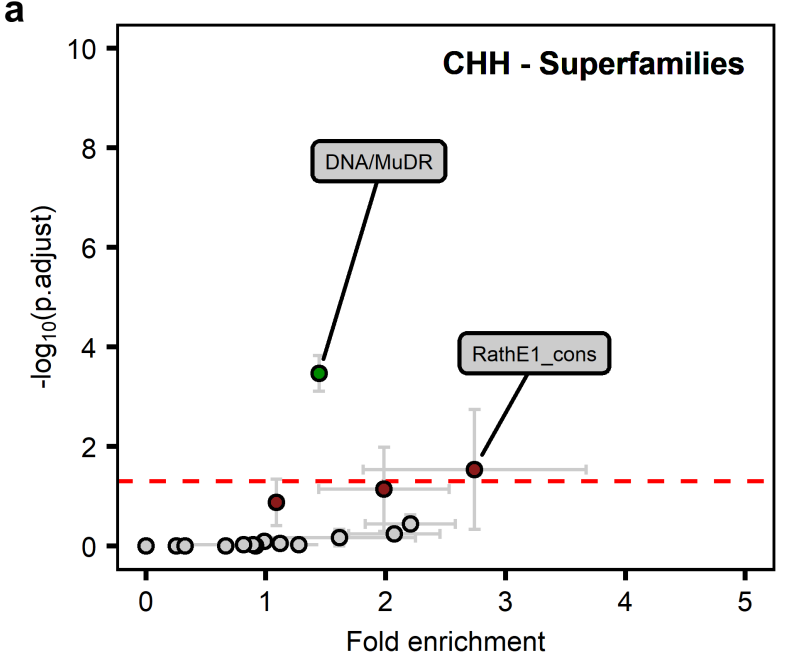

C

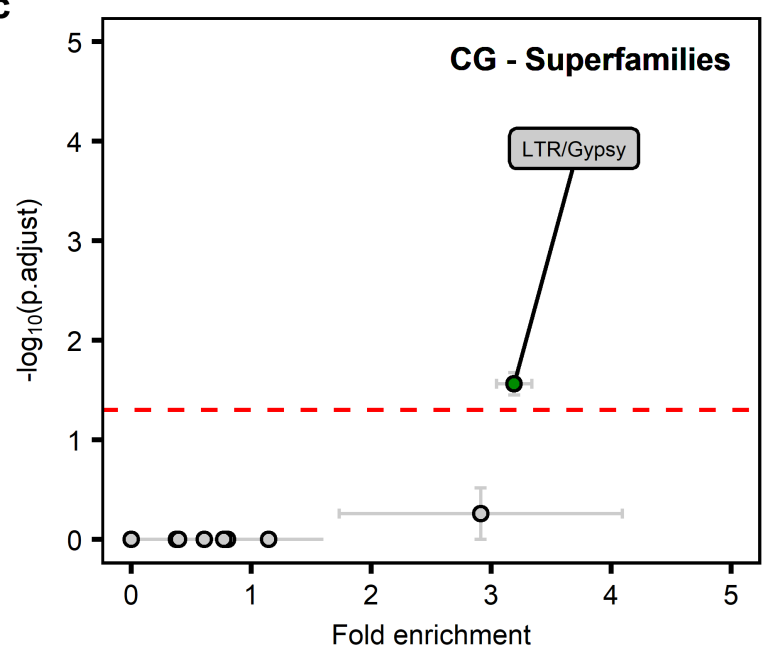

d

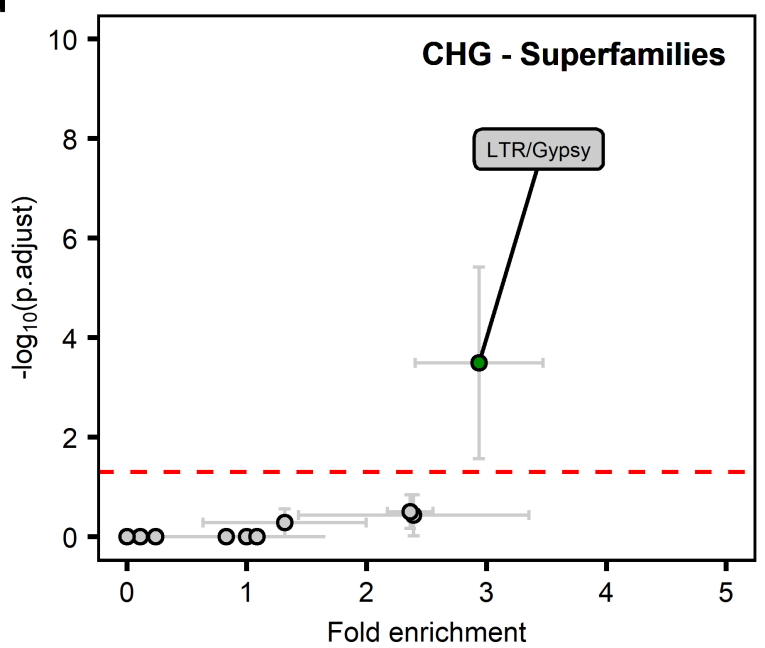

b
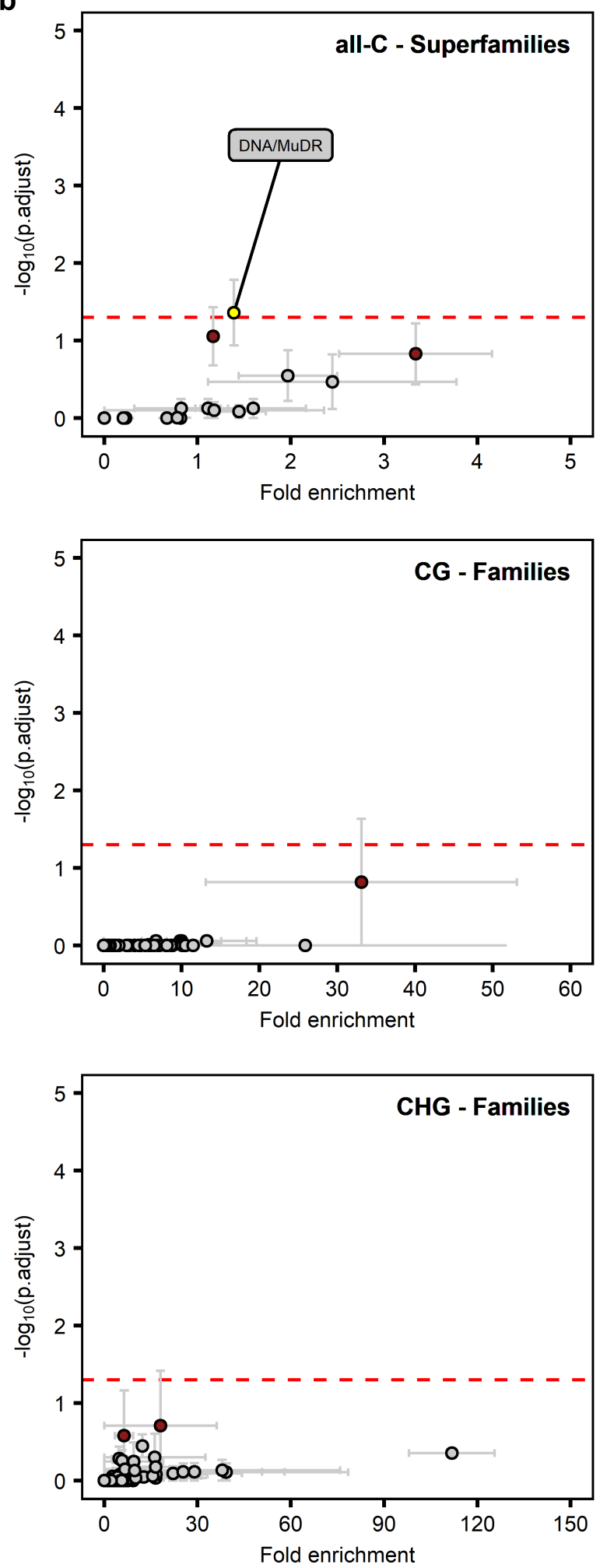

Extended Data Fig. 8 | Transposable element (super)families enriched with JA-

induced differentially methylated regions. Shown are transposable element (TE)

1194 (super)families enriched with JA-induced differentially methylated regions (DMRs) at CHH

(a), all-C (b), CG (c) and CHG (d) sequence contexts. For details about DMR selection, see 
1196 legend to Fig. 5. Enriched TE families for $\mathrm{CHH}$ and all-C contexts are displayed in Fig. 5d,e.

1197 Graphs plot statistical significance against the corresponding fold-enrichment, represented

1198 by mean $-\log _{10}($ p.adj) values $( \pm$ SEM) and mean fold enrichment values $( \pm$ SEM),

1199 respectively. Enrichment is expressed relative to the background of all genome annotated

1200 TEs (TAIR v10). Labelled data points indicate TE (super)families with a mean - $\log _{10}(p . a d j)>$

$1201-\log _{10}(0.05)$ (red dashed line). Brightly coloured data points indicate TE (super)families that

1202 were significantly ( $p . a d j \leq 0.05)$ overrepresented in 1 (red), 2 (yellow) or 3 (green)

1203 comparisons, respectively. 


\section{Wilkinson et al}

1204 SUPPLEMENTARY TABLES

1205 Supplementary Table 1 | Ingredients of the Spodoptera littoralis diet.

1206 Supplementary Table 2 | RT-qPCR primers. 


\section{SUPPLEMENTARY DATA}

1208 Supplementary Data 1 | mRNA-seq read counts for genes displaying an altered response 1209 to JA challenge as a result of prior JA seedling treatment.

1210 Supplementary Data 2 | mRNA-seq read counts for the 832 genes selected by the criteria 1211 (W_JA $>$ W_W $)$ and $\left(J A \_J A>\right.$ W_JA).

1212 Supplementary Data 3 | GO terms that are statistically enriched in gene clusters I-IV of 1213 Extended Data Fig. 3a.

1214 Supplementary Data 4 | mRNA-seq read counts for the 203 genes associated with long1215 term JA-IR against SI.

1216 Supplementary Data 5 | GO terms that are statistically enriched among the 203 genes 1217 associated with long-term JA-IR against SI.

1218 Supplementary Data 6 | mRNA-seq read counts for the 904 genes selected by the criteria 1219 $($ W_JA $<$ W_W $)$ and $\left(J A \_J A<W \_J A\right)$.

Supplementary Data 7 | GO terms that are statistically enriched in gene clusters V-VII of Extended Data Fig. 3a.

Supplementary Data 8 | mRNA-seq read counts for the 796 genes associated with longterm JA-IS to Pst.

Supplementary Data 9 | GO terms that are statistically enriched among the 796 genes associated with long-term JA-IS to Pst.

Supplementary Data 10 | mRNA-seq read counts for the 395 genes selected by the criteria $($ W_JA $>$ W_W $)$ and $\left(J A \_J A<\right.$ W_JA $)$.

Supplementary Data 11 | GO terms that are statistically enriched in gene clusters VIII-X of 1229 Extended Data Fig. 3a. 
1230 Supplementary Data 12 | mRNA-seq read counts for the 144 genes associated with long1231 term JA-IS to $P C$.

1232 Supplementary Data 13 | GO terms that are statistically enriched among the 144 genes 1233 associated with long-term JA-IS to PC.

1234 Supplementary Data 14 | Transcription factor DNA binding motifs that are overrepresented 1235 within promoters of the 203 genes associated with long-term JA-IR to SI.

1236 Supplementary Data 15 | DMR locations and statistics.

1237 Supplementary Data 16 | DMR summary statistics.

1238 Supplementary Data 17 | Consensus DMRs.

1239 Supplementary Data 18 | Raw read data and alignment statistics of the mRNA sequencing 1240 analysis.

1241 Supplementary Data 19 | Raw read data and alignment statistics of the whole genome 1242 bisulfite sequencing analysis. 


\section{Supplementary Files}

This is a list of supplementary files associated with this preprint. Click to download.

- rs148432suppTables.pdf

- suppdata01.xlsx

- suppdata02.xlsx

- suppdata03.xlsx

- suppdata04.xlsx

- suppdata05.xlsx

- suppdata06.xlsx

- suppdata07.xlsx

- suppdata08.xlsx

- suppdata09.xlsx

- suppdata10.xlsx

- suppdata11.xlsx

- suppdata12.xlsx

- suppdata13.xlsx

- suppdata14.xlsx

- suppdata15.xlsx

- suppdata16.xlsx

- suppdata17.xls

- suppdata18.xIsx

- suppdata19.xlsx 